\title{
A Generalized Fast Marching Method for dislocation dynamics
}

\author{
Elisabetta Carlini, Nicolas Forcadel, ${ }^{2}$ Régis Monneau ${ }^{3}$
}

September 11, 2009

\begin{abstract}
In this paper, we consider a Generalized Fast Marching Method (GFMM) as a numerical method to compute dislocation dynamics. The dynamics of a dislocation hyper-surface in $\mathbb{R}^{N}$ (with $N=2$ for physical applications) is given by its normal velocity which is a non-local function of the whole shape of the hyper-surface itself. For this dynamics, we show a convergence result of the GFMM as the mesh size goes to zero. We also provide some numerical simulations in dimension $N=2$.
\end{abstract}

AMS Classification: 65M06, 65M12, 49L25.

Keywords: Hamilton-Jacobi equations, fast marching scheme, convergence, viscosity solutions, dislocation dynamics, non-local equations.

\section{Introduction}

In this paper, we present a numerical approach, called Generalized Fast Marching Method (GFMM), to compute the dynamics of a dislocation. Dislocations are line defects in crystal and their motion is at the origin of plastic properties of metals (we refer to Alvarez et al. [4] for more details on dislocations and for a description of the mathematical model). A smooth dislocation can be represented as the boundary of an open set $\Omega_{t}$ (where $t$ represents the time dependence). The evolution equation can then be written on the characteristic function of $\Omega_{t}$, namely $\theta(x, t)$ which is defined by

$$
\theta(x, t)=\left\{\begin{array}{lll}
1 & \text { if } & x \in \Omega_{t} \\
-1 & \text { if } & x \notin \Omega_{t}
\end{array}\right.
$$

Moreover, $\theta$ is assumed to satisfy the following non-local equation (see Alvarez et al. [4] for an equivalent formulation)

$$
\left\{\begin{array}{lll}
\theta_{t}=\left(\left(c_{0} \star \theta(\cdot, t)\right)(x)+c_{1}(x, t)\right)|D \theta| & \text { in } & \mathbb{R}^{N} \times(0,+\infty), \\
\theta(\cdot, 0)=1_{\Omega_{0}}-1_{\Omega_{0}^{c}} & \text { on } & \mathbb{R}^{N} .
\end{array}\right.
$$

Here and throughout the paper, $\theta_{t}$ denotes the time derivative of $\theta, D \theta$ its gradient in space, $c_{1}$ is a given function, $c_{0}=c_{0}(x)$ is a given kernel and $\star$ denotes the convolution in space.

In $[2,3]$, the authors studied numerically a level set formulation of (1.1). They proposed a convergent scheme and proved a Crandall-Lions [7] type error estimate. Nevertheless, the level set formulation is not completely satisfactory for numerical simulations for dislocation dynamics. This comes from the fact that, on the dislocation line, the gradient of the level set function can get very small very quickly, which creates some difficulties to localize precisely the numerical level set. In particular, this creates numerical oscillations of the front as we point out in Section 8 (Test 2, Figure 5).

For the previous reasons, in the present paper we want to replace the level set method by another method which avoids the difficulty of vanishing gradients. A first attempt in that direction was introduced in [9], and

\footnotetext{
${ }^{1}$ Dipartimento di Matematica, Università di Roma "La Sapienza", P. Aldo Moro, 2, 00185 Roma

${ }^{2}$ CEREMADE, UMR CNRS 7534, Université Paris-Dauphine, Place de Lattre de Tassigny, 75775 Paris Cedex 16 , France

${ }^{3}$ Université Paris-Est, Cermics, Ecole des ponts, 6-8 avenue Blaise Pascal, 77455 Marne la Vallée Cedex 2, France.
} 
was one of our the main motivations to develop a new theory, whose the conclusion is the present paper. The method we consider here is the Generalized Fast Marching Method (GFMM). This algorithm can be seen as an extension of the well-known Fast Marching Method (FMM), introduced independently by Sethian [10, 11] and Tsitsiklis [12]. The classical FMM is a single pass scheme to compute the solution of a stationary eikonal equation with a positive and independent on time velocity $c(x)$. The solution computed gives the arrival time of the front propagating with normal velocity $c(x)$. Recently in [13], this method has been extended to the case of positive velocities $c(x, t)$ depending also on time. Later in [6], a generalization to the case of sign changing velocities $c(x, t)$ has been proposed. Precisely, a new scheme has been designed to compute numerically the discontinuous solution of the following evolutive local equation

$$
\left\{\begin{array}{lll}
\theta_{t}=c(x, t)|D \theta| & \text { in } & \mathbb{R}^{N} \times(0,+\infty), \\
\theta(\cdot, 0)=1_{\Omega_{0}}-1_{\Omega_{0}^{c}} & \text { on } & \mathbb{R}^{N}
\end{array}\right.
$$

In this problem the velocity $c(x, t)$ is assumed to be a given function (contrarily to equation (1.1)). Here the evolution of the front is given by the evolution of the interface between the two phases of $\theta$ and then the problem of the vanishing gradient is automatically solved.

Even if an evolution equation is approximated by the GFMM, in the case where the velocity $c(x, t)$ is Lipschitz in time (with a reasonable Lipschitz constant), the computational complexity is $O(M \log (M))$, where $M$ is the total number of grid points, as in the classical FMM (see [6]).

On the one hand, the main result of [6] is a convergence result of the method as the mesh size goes to zero. On the other hand, one drawback of the scheme proposed in [6] is the fact that the method is not completely monotone, which makes the analysis difficult for the application of this scheme to non-local equations like for dislocation dynamics. One major progress has been very recently given in [8], where an improvement of the GFMM has been proposed with a similar computational complexity $O(M \log (M))$. This new version of the GFMM is indeed truly monotone, and this is this version of GFMM that we will use in this paper to perform our analysis. Let us mention that one originality of this scheme is that several different notions of times can be defined at the same point of the front for the same step of the algorithm.

The main result that we get in the present paper is the following (presented here in very vague terms, see Theorem 3.4 for a precise version):

Theorem (Convergence of the numerical method)

Under suitable assumptions, the numerical solution given by a suitable GFMM, is convergent as the mesh size goes to zero, to the solution $\theta$ of equation (1.1) of dislocation dynamics.

This shows in particular that a GFMM can be considered as a good alternative to the level set method. This conclusion is moreover supported by the numerical simulations presented at the end of the paper, which are compared to analogous simulations obtained using the level set method (see Test 2).

\section{Organization of the paper}

In Section 2, we first recall the monotone GFMM given in [8] for the local equation and then we give its extension to compute the numerical solution of the non-local equation of dislocation dynamics. In Section 3 we present our main results about the convergence of the numerical method to the solution of the non-local equation. To prove the results we will use some known properties (monotonicity, convergence) about the GFMM presented in [8] for local equations. These properties are recalled in Section 4.

In Section 5 we prove the general convergence result and in the two following sections, we apply the result in two particular cases: smooth evolutions (Lipschitz curves in $\mathbb{R}^{2}$ ) with sign changing (non-local) velocities in Section 6 and non smooth evolutions (with the condition of interior ball in $\mathbb{R}^{N}$ ) with positive (non-local) velocities in Section 7.

In Section 8, we provide some numerical simulations for the non-local equation describing dislocation dynamics. Finally, in Appendix A, we give the precise version of the GFMM, which has been used to perform the numerical simulations of Section 8. This version is not monotone in the general case of speed changing sign in time, but it is simpler to implement in practice than the one presented in Section 2 .

\section{GFMM algorithms for local and non-local equations}

\subsection{A monotone GFMM for the local equation}

We present here the monotone GFMM algorithm studied in [8] (which appears to be a modified version of the GFMM algorithm introduced in [6]). 


\subsubsection{Preliminaries}

Let us consider a lattice $Q=\left\{x_{I}=\left(x_{i_{1}}, . ., x_{i_{N}}\right)=\left(i_{1} \Delta x, . ., i_{N} \Delta x\right), I=\left(i_{1}, . ., i_{N}\right) \in \mathbb{Z}^{N}\right\}$ with space step $\Delta x$. We will also use a time step $\Delta t>0$ (which does not satisfy any CFL condition).

The following definitions will be useful in the following.

Definition 2.1 The neighbourhood of the node $I \in \mathbb{Z}^{N}$ is the set

$$
V(I) \equiv\left\{J \in \mathbb{Z}^{N}:|J-I| \leq 1\right\} .
$$

Definition 2.2 The numerical boundary $\partial E$ of a set $E \subset \mathbb{Z}^{N}$ is

$$
\partial E \equiv V(E) \backslash E
$$

with

$$
V(E)=\bigcup_{I \in E} V(I)
$$

Definition 2.3 Given a field $\theta_{I}^{n}$ with values +1 and -1 , we define the two phases

$$
\Theta_{ \pm}^{n} \equiv\left\{I: \theta_{I}^{n}= \pm 1\right\}
$$

and the fronts

$$
F_{ \pm}^{n} \equiv \partial \Theta_{\mp}^{n}, \quad F^{n} \equiv F_{+}^{n} \cup F_{-}^{n} .
$$

As in $[6,8]$, we have to "regularize" the numerical velocity in space in order to avoid the duplication of the front.

Definition 2.4 Given the speed $c_{I}^{n} \equiv c\left(x_{I}, t_{n}\right)$ we define the function

$$
\widehat{c}_{I}^{n} \equiv \begin{cases}0 & \text { if there exists } J \in V(I) \text { such that }\left(c_{I}^{n} c_{J}^{n}<0 \text { and }\left|c_{I}^{n}\right| \leq\left|c_{J}^{n}\right|\right), \\ c_{I}^{n} & \text { otherwise. }\end{cases}
$$

We now describe the monotone GFMM algorithm for unsigned velocity. As in the classical FMM, we define the Narrow Band (NB) which consists in the points $I \in \mathbb{Z}^{N}$ that can be immediately reached by the front:

$$
N B^{n}=\left\{I \in \mathbb{Z}^{N}, \exists J \in V(I), \theta_{I}^{n}=-\theta_{J}^{n} \text { and } \theta_{I}^{n} \hat{c}_{I}^{n}<0\right\}, \quad N B_{ \pm}^{n}=N B^{n} \cap\left\{I, \theta_{I}^{n}= \pm 1\right\}
$$

We observe that the Narrow Bands $N B_{ \pm}^{n}$ are related with the previous definition of fronts set as follows:

$$
N B_{+}^{n}=F_{+}^{n} \cap\left\{I, \hat{c}_{I}^{n}<0\right\}, \quad N B_{-}^{n}=F_{-}^{n} \cap\left\{I, \hat{c}_{I}^{n}>0\right\} .
$$

Fig. 1 shows the Front and the Narrow Band, the white part of the picture represents the domain where the speed is negative and the gray part the domain with positive speed.

As in the FMM, for all $I \in N B^{n}$, we have to compute a tentative value (which we denote $\tilde{u}_{I}^{n}$ in the sequel) of the arrival time of the front at point $I$. To compute this tentative value, we define the points that are useful for $I$, i.e. that we will use in the computation of $\tilde{u}_{I}^{n}$ :

$$
\text { for } I \in N B^{n}, \quad \mathcal{U}^{n}(I)=\left\{J \in V(I), \theta_{I}^{n}=-\theta_{J}^{n}\right\}, \quad \mathcal{U}^{n}=\bigcup_{I \in N B^{n}} \mathcal{U}^{n}(I)
$$

For all the points $J$ that are useful for a point $I \in N B^{n}$ (i.e. $J \in \mathcal{U}^{n}(I)$ ) we will introduce a time $u_{J \rightarrow I}^{n}$. This time $u_{J \rightarrow I}^{n}$ can be interpreted as the time when the front $F^{n}$ starts to go from point $J$ to point $I$ and will be used to compute the tentative value at point $I$. One originality of the GFMM that we consider here, is that we may have $u_{J \rightarrow I}^{n} \neq u_{J \rightarrow K}^{n}$ for $I \neq K$ with $I, K \in N B^{n}$. This means that we may need a priori several times at the same point $J \in \mathcal{U}^{n}$. This possibility of multiple values of the time at the same point $J$ is one of the key property to prove that the GFMM algorithm is monotone, see [8] and Section 4. Then the monotonicity property of the GFMM allows us to obtain convergence. 


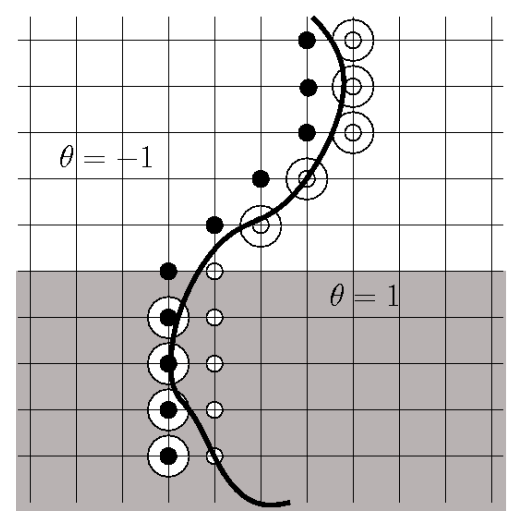

Figure 1: The Front $F^{+}$(white spot), the front $F^{-}$(black spot), the Narrow Band $N B^{+}$(circled white spot) and the Narrow Band $N B^{-}$(circled black spot). In the white domain the speed is negative, in the gray domain the speed is positive.

Once we have computed the tentative value for all points of the Narrow Band, we denote by $\tilde{t}_{n}$ the minimum of all these values. Unfortunately, this sequence of "candidate" time is not necessarily nondecreasing, so we have to truncate $\tilde{t}_{n}$ to define $t_{n}$ such that (see Step 6 of the algorithm):

$$
0 \leq t_{n}-t_{n-1} \leq \Delta t
$$

for a fixed $\Delta t$ (independent of $\Delta x$ ). The algorithm is now very similar to the classical Fast Marching Method. We accept (in most of the cases, see Step 7 of the algorithm) all the points that realize the minimum (i.e., we change the value of the $\theta$ ), we update the Narrow Band $N B^{n}$ and the useful front $\mathcal{U}^{n}$, then we redefine the value $u_{J \rightarrow I}^{n}$ and we iterate.

\subsubsection{The monotone GFMM step-by-steps}

We now give the precise formulation of the algorithm:

\section{Initialization}

1. Set $n=1$

2. Initialize the field $\theta^{0}$ as $\theta_{I}^{0}=\left\{\begin{aligned} 1 & \text { for } x_{I} \in \Omega_{0}, \\ -1 & \text { elsewhere }\end{aligned}\right.$

3. Initialize the time for points I

$$
u_{I \rightarrow K}^{0}= \begin{cases}t_{0}=0 & \text { if } K \in N B^{0} \text { and } I \in \mathcal{U}^{0}(K) \\ +\infty & \text { otherwise }\end{cases}
$$

\section{Loop}

4. Compute $\tilde{u}^{n-1}$ on $N B^{n-1}$

Let $I \in N B^{n-1}$, then we compute $\tilde{u}_{I}^{n-1}$ as the solution of the following quadratic equation:

$$
\sum_{k=1}^{N}\left(\max _{ \pm}\left(0, \tilde{u}_{I}^{n-1}-u_{I^{k, \pm} \rightarrow I}^{n-1}\right)\right)^{2}=\frac{(\Delta x)^{2}}{\left|\widehat{c}_{I}^{n-1}\right|^{2}}
$$

where

$$
I^{k, \pm}=\left(i_{1}, . ., i_{k-1}, i_{k} \pm 1, i_{k+1}, . ., i_{N}\right) .
$$

5. $\tilde{t}_{n}=\min \left\{\tilde{u}_{I}^{n-1}, I \in N B^{n-1}\right\}$. 
6. Truncate $\tilde{t}_{n}$ $t_{n}=\max \left(t_{n-1}, \min \left\{\tilde{t}_{n}, t_{n-1}+\Delta t\right\}\right)$

7. if $t_{n}<\tilde{t}_{n}$ go to 4 with $n:+1, \theta^{n}=\theta^{n-1}$ and $u^{n}=u^{n-1}$.

8. Initialize the new accepted points $N A_{ \pm}^{n}=\left\{I \in N B_{ \pm}^{n-1}, \tilde{u}_{I}^{n-1}=\tilde{t}_{n}\right\}, N A^{n}=N A_{+}^{n} \cup N A_{-}^{n}$

9. Reinitialize $\theta^{n}$

$$
\theta_{I}^{n}=\left\{\begin{aligned}
-\theta_{I}^{n-1} & \text { for } I \in N A^{n} \\
\theta_{I}^{n-1} & \text { elsewhere }
\end{aligned}\right.
$$

10. Reinitialize $u_{I \rightarrow K}^{n}$

$$
u_{I \rightarrow K}^{n}= \begin{cases}\min \left(u_{I \rightarrow K}^{n-1}, t_{n}\right) & \text { if } K \in N B^{n} \text { and } I \in \mathcal{U}^{n}(K) \\ +\infty & \text { otherwise }\end{cases}
$$

11. Set $n:+1$ and go to 4

Remark 2.5 We refer to [6, Subsection 2.3] for a detailed discussion on the complexity and on the implementation of an algorithm very similar to the present one. The only difference is that in the present algorithm we may have local times $u_{I \rightarrow J}$ for each neighbour $J \in V(I) \backslash\{I\}$, which in the worse case may introduce a factor $2 N$ (the number of nearest neighbours of a point in $\mathbb{Z}^{N}$ ) in the computation of the total complexity of the algorithm.

\subsubsection{Reconstruction of the numerical solution}

We now explain how to build the numerical solution, using the output of the monotone GFMM algorithm.

We first remark that the physical sequence of time $\left\{t_{n}, n \in \mathbb{N}\right\}$, defined at the Step 6 of the algorithm is non-decreasing and we can extract a subsequence $\left\{t_{n_{k}}, k \in \mathbb{N}\right\}$ strictly increasing such that

$$
t_{n_{k}}=t_{n_{k}+1}=\ldots=t_{n_{k+1}-1}<t_{n_{k+1}} .
$$

Then we denote by $S_{I}^{k}$ the square cell $S_{I}^{k}=\left[x_{I}, x_{I}+\Delta x\left[\times\left[t_{n_{k}}, t_{n_{k+1}}[\right.\right.\right.$ with

$$
\left[x_{I}, x_{I}+\Delta x\left[=\Pi_{\alpha=1}^{N}\left[x_{i_{\alpha}}, x_{i_{\alpha}}+\Delta x[\right.\right.\right.
$$

and by $\varepsilon$ the couple

$$
\varepsilon=(\Delta x, \Delta t)
$$

We now define the following function:

$$
\theta^{\varepsilon}(x, t)=\theta_{I}^{n_{k+1}-1} \text { if }(x, t) \in S_{I}^{k} .
$$

which can be interpreted as the numerical approximation of the solution $\theta$ of the local equation (1.2), reconstructed using the monotone GFMM.

\subsection{A non-local GFMM algorithm}

Our aim is to adapt the previous algorithm to compute a numerical approximation of a solution of the non-local equation (1.1).

Given a data $\theta=\left(\theta_{I}\right)_{I \in \mathbb{Z}^{N}}$, we define its extension on $\mathbb{R}^{N}$ by

$$
\theta^{\Delta}(x)=\sum_{I \in \mathbb{Z}^{N}} \theta_{I} 1_{\left[x_{I}, x_{I}+\Delta x[\right.}(x)
$$

and the corresponding non-local velocity by

$$
c\left[\theta^{\Delta}\right](x, t)=\left(c_{0} \star \theta^{\Delta}\right)(x)+c_{1}(x, t)
$$

The non-local GFMM algorithm is then defined from the local GFMM, adding an initializing step before the main cycle and adding a new step before Step 11: 
3b. Initialize the speed: $\quad c_{I}^{0} \equiv c\left[\left(\theta^{0}\right)^{\Delta}\right]\left(x_{I}, t_{0}\right)$, with $t_{0}=0$ and $p:=0$.

10b. Compute the speed $c_{I}^{n}$ :

Given $t_{n-1} \in[p \Delta T,(p+1) \Delta T)$ for some $p \in \mathbb{N}$, we set

$$
c_{I}^{n} \equiv \begin{cases}c_{I}^{n-1} & \text { if } \quad t_{n}<(p+1) \Delta T \\ c\left[\left(\theta^{n-1}\right)^{\Delta}\right]\left(x_{I}, t_{n}\right) & \text { if } \quad t_{n} \geq(p+1) \Delta T\end{cases}
$$

If $t_{n} \geq(p+1) \Delta T$, set $p:=p+q^{*}$ with $q^{*}=\sup \left\{q, t_{n} \geq(p+q) \Delta T\right\}$.

Here $\Delta T>0$ is a time step that has been introduced to avoid to recompute the non-local velocity at each step of the algorithm. This time step $\Delta T$ is not connected to the time step $\Delta t$ (nor to the space step $\Delta x)$. Indeed, we can choose $\Delta t \ll \Delta T \ll 1$ or for instance $\Delta t>\Delta T$.

When we deal with the non-local equation, we redefine the parameter of discretization $\varepsilon$ as

$$
\varepsilon=(\Delta x, \Delta t, \Delta T)
$$

that will be required to converge to zero, to insure the convergence of the numerical method in that case.

Remark 2.6 Indeed, we could choose " $\Delta T=0$ ", which means to recompute the velocity at each iteration of the algorithm such that $t_{n}>t_{n-1}$.

Remark 2.7 The fact that we use $\theta^{n-1}$ to recompute the velocity at time $t_{n}$ (even if we know $\theta^{n}$ ) could seem curious. The reason is that, once we have computed the solution $\left(\theta_{I}^{n}\right)_{n, I}$ to the non-local GFMM and then constructed $\theta^{\varepsilon}$, it is possible to interpret the non-local GFMM as the local GFMM with a velocity which is now precomputed using the solution $\theta^{\varepsilon}$ only.

More technically, this is due to the fact that we can write (see (5.3)) the velocity as a convolution with the function $\theta^{\varepsilon}$. Indeed, let us consider a sequence $\left\{n_{k}, k \in \mathbb{N}\right\}$ with the following time iterations

$$
t_{n_{k}}=t_{n_{k}+1}=\ldots=t_{n_{k+1}-1}<t_{n_{k+1}} .
$$

Since $t_{n} \geq(p+1) \Delta T$ and $t_{n-1}<(p+1) \Delta T$, then $n-1=n_{k+1}-1$ for some index $k$. Using now the definition (2.3), we get

$$
\theta^{\varepsilon}(x, t)=\theta_{I}^{n_{k+1}-1}=\theta_{I}^{n-1} \text { if }(x, t) \in S_{I}^{k} .
$$

and this fact allows us to write the speed as in (5.3):

$$
c_{I}^{n}=c\left[\theta^{\varepsilon}\left(\cdot, t_{n_{k}}\right)\right]\left(x_{I}, t_{n_{k+1}}\right) .
$$

\section{Main results}

We consider an open set $\Omega_{0} \subset \mathbb{R}^{N}$ and the non-local eikonal equation

$$
\left\{\begin{array}{l}
\theta_{t}(x, t)=c[\theta(\cdot, t)](x, t)|D \theta(x, t)| \quad \text { on } \quad \mathbb{R}^{N} \times(0,+\infty) \\
\theta(\cdot, 0)=1_{\Omega_{0}}-1_{\Omega_{0}^{c}}
\end{array}\right.
$$

where

$$
c[\theta(\cdot, t)](x, t)=\left(c_{0} \star \theta(\cdot, t)\right)(x)+c_{1}(x, t) .
$$

We make the following assumption:

(A1) Regularity of the velocity:

$$
c_{1} \in W^{1, \infty}\left(\mathbb{R}^{N} \times[0,+\infty)\right), c_{0} \in W^{1,1}\left(\mathbb{R}^{N}\right) \cap L^{\infty}\left(\mathbb{R}^{N}\right) .
$$

We denote by $L_{c_{1}}$ the time Lipschitz constant of $c_{1}$ such that

$$
\left|c_{1}\left(x, t^{\prime}\right)-c_{1}(x, t)\right| \leq L_{c_{1}}\left|t^{\prime}-t\right| \quad \text { for any } \quad x \in \mathbb{R}^{N}, \quad t^{\prime}, t \geq 0
$$




\subsection{First main results}

Then our first main result concerns the case of a dislocation curve in dimension $N=2$, for which a short time existence and uniqueness result is known (see [4]).

Theorem 3.1 (Dislocation loop for short time with non signed velocity)

Assume (A1) and that $\Omega_{0} \subset \mathbb{R}^{2}$ is a $C^{3}$ bounded connected and simply connected open set. Then there exists $T^{*}>0$ such that for $\varepsilon=(\Delta x, \Delta t, \Delta T)$ converging to zero, we have

$$
\theta^{\varepsilon} \rightarrow \theta \quad \text { in } L^{\infty}\left(\left[0, T^{*}\right) ; L^{1}\left(\mathbb{R}^{N}\right)\right)
$$

where $\theta^{\varepsilon}$ is given in (2.3) using the non-local GFMM algorithm given in Subsection 2.2 and $\theta$ is the unique solution of (3.1) on the time interval $\left[0, T^{*}\right)$.

In higher dimensions, in the case where the non-local velocity is positive, a global existence result is known under suitable assumptions (see [1]). In that case, we get the following result

Theorem 3.2 (Large time dislocation dynamics with nonnegative velocity) Assume that $c_{0} \in W^{2,1}\left(\mathbb{R}^{N}\right) \cap L^{\infty}\left(\mathbb{R}^{N}\right), c_{1} \in W^{2, \infty}\left(\mathbb{R}^{N} \times[0,+\infty)\right)$ and $c_{1}(x, t) \geq\left|c_{0}\right|_{L^{1}\left(\mathbb{R}^{N}\right)}+\delta$ with $\delta>0$. Assume also that $\Omega_{0} \subset \mathbb{R}^{N}$ is a bounded $C^{2}$ open set. Then for $\varepsilon=(\Delta x, \Delta t, \Delta T)$ converging to zero, we have

$$
\theta^{\varepsilon} \rightarrow \theta \quad \text { in } L^{\infty}\left([0,+\infty) ; L^{1}\left(\mathbb{R}^{N}\right)\right)
$$

where $\theta^{\varepsilon}$ is given in (2.3) using the non-local GFMM algorithm given in Subsection 2.2 and $\theta$ is the unique solution of (3.1).

\subsection{A general convergence result}

In this subsection, we will present a general convergence result whose Theorems 3.1 and 3.2 will be seen as consequences. To this end, we recall the following definition, of upper semi-continuous envelope

$$
\theta^{*}(x, t)=\limsup _{(y, s) \rightarrow(x, t)} \theta(y, s)
$$

and of lower semi-continuous envelope

$$
\theta_{*}(x, t)=\liminf _{(y, s) \rightarrow(x, t)} \theta(y, s) .
$$

Then we introduce the following assumptions:

(A2) Existence, uniqueness and Lipschitz in time non local velocity:

There exists a finite time $T^{*}>0$ and a unique solution $\theta \in C\left(\left[0, T^{*}\right), L^{1}\left(\mathbb{R}^{N}\right)\right)$ which is a discontinuous viscosity solution of equation (3.1). Moreover, the velocity is Lipschitz in time, i.e.

$$
\left|c\left[\theta\left(\cdot, t^{\prime}\right)\right]\left(x, t^{\prime}\right)-c[\theta(\cdot, t)](x, t)\right| \leq L_{c}\left|t^{\prime}-t\right|
$$

for any $x \in \mathbb{R}^{N}$ and $t^{\prime}, t \in\left[0, T^{*}\right)$

Remark 3.3 Here the uniqueness of the discontinuous viscosity solution $\theta$ has to be understood as the fact that all the solutions has the same upper (resp. lower) semi-continuous envelopes.

(A3') $L^{1}$ stability estimate for a perturbed local problem:

For a given local velocity $c \in W^{1, \infty}\left(\mathbb{R}^{N} \times\left[0, T^{*}\right)\right)$, there exists $e_{0}>0$, such that for any function $e \in C\left(\left[0, T^{*}\right)\right)$ satisfying $|e|_{L^{\infty}\left(\left[0, T^{*}\right)\right)} \leq e_{0}$, the solution $\theta^{e}$ of the following perturbed problem,

$$
\begin{cases}\theta_{t}^{e}(x, t)=c^{e}(x, t)\left|D \theta^{e}(x, t)\right| & \text { on } \mathbb{R}^{N} \times\left(0, T^{*}\right) \text { with } c^{e}(x, t)=c(x, t)+e(t) \\ \theta^{e}(\cdot, 0)=1_{\Omega_{0}}-1_{\Omega_{0}^{c}} & \text { on } \mathbb{R}^{N},\end{cases}
$$

exists, is unique and satisfies

$$
\left|\left(\theta^{e}\right)^{*}(\cdot, t)-\left(\theta^{e}\right)_{*}(\cdot, t)\right|_{L^{1}\left(\mathbb{R}^{N}\right)}=0 \quad \text { for any } \quad 0 \leq t<T^{*} .
$$


We also assume that there exists some constants $C_{0}>0, \bar{T}>0$ such that for every $0 \leq T_{0}<T^{*}$, if moreover $\left|\left(\theta^{e}-\theta\right)\left(\cdot, T_{0}\right)\right|_{L^{1}\left(\mathbb{R}^{N}\right)} \leq e_{0}$, then

$$
\left|\theta^{e}-\theta\right|_{L^{\infty}\left(\left[T_{0}, T_{0}+T\right) ; L^{1}\left(\mathbb{R}^{N}\right)\right)} \leq C_{0}\left(|e|_{L^{\infty}\left(\left[T_{0}, T_{0}+T\right)\right)} T+\left|\left(\theta^{e}-\theta\right)\left(\cdot, T_{0}\right)\right|_{L^{1}\left(\mathbb{R}^{N}\right)}^{\frac{1}{N}}\right)
$$

for any $0<T \leq \min \left(\bar{T}, T^{*}-T_{0}\right)$.

(A3) $L^{1}$ stability estimate for a perturbed non local problem:

For $\theta$ the solution given in assumption (A2), we assume that (A3') holds with the velocity $c(x, t):=$ $c[\theta(\cdot, t)](x, t)$.

Theorem 3.4 (General convergence result for non local dynamics)

Let us consider problem (3.1) with a $C^{2}$ bounded open set $\Omega_{0} \subset \mathbb{R}^{N}$, under assumptions (A1)-(A2)-(A3). Then for $\varepsilon=(\Delta x, \Delta t, \Delta T)$ converging to zero, we have

$$
\theta^{\varepsilon} \rightarrow \theta \quad \text { in } L^{\infty}\left(\left[0, T^{*}\right) ; L^{1}\left(\mathbb{R}^{N}\right)\right)
$$

where $\theta^{\varepsilon}$ is given in (2.3) using the non-local GFMM algorithm given in Subsection 2.2 and $\theta$ is the unique solution of (3.1) on the time interval $\left[0, T^{*}\right)$.

\section{Preliminary results for the monotone GFMM for the local equa- tion}

In this Section, we recall some key properties of the monotone GFMM for the local equation (for which we refer the reader to [8]) and also present some consequences.

The following result is stated in Theorem 3.4 of [8].

\section{Theorem 4.1 (Comparison principle for the $\theta^{\varepsilon}$ )}

Let $T>0$. Let two velocities $c_{u}$ and $c_{v}$. Given $\theta_{u, J}^{0}\left(\right.$ resp. $\left.\theta_{v, J}^{0}\right)$ for all $J \in \mathbb{Z}^{N}$, we denote by $\left(t_{n_{k}}\right)$ and $\left(s_{m_{p}}\right)$ the increasing sequences of times defined as in (2.2) for the GFMM associated to $c_{u}$ and $c_{v}$ respectively. We assume that for all $t_{n_{k}}<T, s_{m_{p}}<T$, such that $\left[t_{n_{k}}, t_{n_{k+1}}\right) \cap\left[s_{m_{p}}, s_{m_{p+1}}\right) \neq \emptyset$, then the two velocities satisfy for all $x \in \mathbb{R}^{N}$

$$
c_{v}\left(x, s_{m_{p}}\right) \geq c_{u}\left(x, t_{n_{k}}\right) .
$$

If $\theta_{v, J}^{0} \geq \theta_{u, J}^{0}$ for all $J \in \mathbb{Z}^{N}$, then

$$
\theta_{v}^{\varepsilon}(x, t) \geq \theta_{u}^{\varepsilon}(x, t)
$$

for all $(x, t) \in \mathbb{R}^{N} \times[0, T)$.

Remark 4.2 The assumption on the comparison of the velocities is slightly different from the one of [8], but this is in fact what is used in the proof. Indeed, the algorithm used only the velocity at the time of the form $t_{n_{k}}<T$ and $s_{m_{p}}<T$. Hence, it suffices to compare the velocities at those times.

We now give the convergence result for the monotone GFMM (presented in Subsection 2.1). To do this, we have to define the half-relaxed limits of $\theta^{\varepsilon}$ :

$$
\bar{\theta}^{0}(x, t)=\limsup _{\varepsilon \rightarrow 0, y \rightarrow x, s \rightarrow t} \theta^{\varepsilon}(y, s), \quad \underline{\theta}^{0}(x, t)=\liminf _{\varepsilon \rightarrow 0, y \rightarrow x, s \rightarrow t} \theta^{\varepsilon}(y, s) .
$$

Then the following result is stated in Theorem 3.6 of [8].

\section{Theorem 4.3 (Convergence)}

Let $c \in W^{1, \infty}\left(\mathbb{R}^{N} \times\left[0, T^{*}\right)\right.$ ) and $\Omega_{0}$ be a $C^{2}$ bounded open set. Then, $\bar{\theta}^{0}$ (resp. $\left.\underline{\theta}^{0}\right)$ is a viscosity sub-solution (resp. super-solution) of (1.2). In particular, if (1.2) satisfies a comparison principle, then $\bar{\theta}^{0}=\left(\underline{\theta}^{0}\right)^{*}$ and $\left(\bar{\theta}^{0}\right)_{*}=\underline{\theta}^{0}$ is the unique discontinuous viscosity solution of (1.2). 
For simplicity of notation, given a constant $e_{0}>0$, we now define

$$
\mathcal{E}=\left\{e \in W^{1, \infty}\left(\left[0, T^{*}\right)\right),|e|_{W^{1, \infty}\left(\left[0, T^{*}\right)\right)} \leq e_{0}\right\} .
$$

We then have the following result which is a straightening of the convergence result Theorem 4.3 and whose proof is exactly the same and therefore is skipped:

\section{Lemma 4.4 (Uniform convergence)}

Let $\Omega_{0}$ be a $C^{2}$ bounded open set and $c \in W^{1, \infty}\left(\mathbb{R}^{N} \times\left[0, T^{*}\right)\right)$ be such that assumption (A3') holds for $c$. Let us consider two sequences $\left(e_{n}\right)_{n \in \mathbb{N}} \subset \mathcal{E}$ and $\left(\varepsilon_{n}\right)_{n \in \mathbb{N}}$ and a function $e \in \mathcal{E}$ such that $\varepsilon_{n} \rightarrow 0$ and $e_{n} \rightarrow e$ in $L^{\infty}\left(\left[0, T^{*}\right)\right)$ as $n \rightarrow \infty$. We call $\theta^{e}$ the solution of problem (3.4) and $\theta^{\varepsilon_{n}, e_{n}}$ the numerical solution given in (2.3) (with $\varepsilon=\varepsilon_{n}$ ) using the GFMM algorithm ( see Subsection 2.1) with speed $c^{e_{n}}(x, t)=c(x, t)+e_{n}(t)$. Then we have for all $(x, t) \in \mathbb{R}^{N} \times\left[0, T^{*}\right)$

$$
\limsup _{y \rightarrow x, s \rightarrow t, n \rightarrow \infty} \theta^{\varepsilon_{n}, e_{n}}(y, s) \leq\left(\theta^{e}\right)^{*}(x, t) \quad \liminf _{y \rightarrow x, s \rightarrow t, n \rightarrow \infty} \theta^{\varepsilon_{n}, e_{n}}(y, s) \geq\left(\theta^{e}\right)_{*}(x, t) .
$$

Then we have the following result:

\section{Proposition 4.5 (Uniform error estimate for the perturbed local dynamics)}

Let $\Omega_{0}$ be a $C^{2}$ bounded open set and $c \in W^{1, \infty}\left(\mathbb{R}^{N} \times\left[0, T^{*}\right)\right)$ be such that assumption (A3') holds for c. Let $e \in \mathcal{E}$ where the set $\mathcal{E}$ is build with the constant $e_{0}$ given in assumption (A3'). Let $\theta^{e}$ be the solution of (3.4). Let us consider $\theta^{\varepsilon, e}$ the numerical approximation of $\theta^{e}$ given in (2.3) using the GFMM algorithm (see Subsection 2.1) with speed $c^{e}(x, t)=c(x, t)+e(t)$.

Then, there exists a modulus of continuity $\omega_{1}(\varepsilon)$ such that:

$$
\left|\theta^{e}-\theta^{\varepsilon, e}\right|_{L^{\infty}\left(\left[0, T^{*}\right) ; L^{1}\left(\mathbb{R}^{N}\right)\right)} \leq \omega_{1}(\varepsilon) \text { uniformly in } e \in \mathcal{E} .
$$

\section{Proof of Proposition 4.5}

Let us consider two sequences $\left(e_{n}\right)_{n \in \mathbb{N}} \subset \mathcal{E}$ and $\left(\varepsilon_{n}\right)_{n \in \mathbb{N}}$ and a function $e \in \mathcal{E}$ such that $\varepsilon_{n} \rightarrow 0$ and $e_{n} \rightarrow e$ for $n \rightarrow \infty$. We call $\theta^{e}$ the solution of problem (3.4) and $\theta^{\varepsilon_{n}, e_{n}}$ the numerical solution given in (2.3)(with $\varepsilon=\varepsilon_{n}$ ) using the GFMM algorithm (see Subsection 2.1) with speed $c^{e_{n}}(x, t)=c(x, t)+e_{n}(t)$. We will prove that for $n \rightarrow \infty$

$$
\left|\theta^{\varepsilon_{n}, e_{n}}-\theta^{e}\right|_{L^{\infty}\left(\left[0, T^{*}\right) ; L^{1}\left(\mathbb{R}^{N}\right)\right)} \rightarrow 0
$$

We define

$$
\bar{\theta}^{e}(x, t)=\limsup _{y \rightarrow x, s \rightarrow t, n \rightarrow \infty} \theta^{\varepsilon_{n}, e_{n}}(y, s) \quad \text { and } \quad \underline{\theta}^{e}(x, t)=\liminf _{y \rightarrow x, s \rightarrow t, n \rightarrow \infty} \theta^{\varepsilon_{n}, e_{n}}(y, s) .
$$

From Lemma 4.4, we have for all $(x, t) \in \mathbb{R}^{N} \times\left[0, T^{*}\right)$

$$
\left(\theta^{e}\right)_{*}(x, t) \leq \underline{\theta}^{e}(x, t) \leq \bar{\theta}^{e}(x, t) \leq\left(\theta^{e}\right)^{*}(x, t) .
$$

Using Assumption $\left(A 3^{\prime}\right)$, we deduce that for any fixed $t \in\left[0, T^{*}\right)$ and $t_{n} \rightarrow t$, we have for $n \rightarrow \infty$

$$
\left|\theta^{\varepsilon_{n}, e_{n}}\left(x, t_{n}\right)-\theta^{e}\left(x, t_{n}\right)\right| \rightarrow 0 \text { for a.e } x \in \mathbb{R}^{N} .
$$

From the boundedness of $\Omega_{0}$ and the finite velocity property of the eikonal equation, we deduce that there exists $R>0$ (depending on $T^{*}$ ) such that

$$
\operatorname{supp}\left(\theta^{e}\left(\cdot, t_{n}\right)+1\right) \subset B_{R}(0) .
$$

Moreover, using the barrier functions as in [6, Proposition 5.2], we get also (up to increase $R$ independently on $\varepsilon$ and $e$ bounded):

$$
\operatorname{supp}\left(\theta^{\varepsilon_{n}, e_{n}}\left(\cdot, t_{n}\right)+1\right) \subset B_{R}(0) .
$$

Therefore

$$
\operatorname{supp}\left(\theta^{\varepsilon_{n}, e_{n}}\left(\cdot, t_{n}\right)-\theta^{e}\left(\cdot, t_{n}\right)\right) \subset B_{R}(0)
$$

and since $\left|\theta^{\varepsilon_{n}, e_{n}}-\theta^{e}\right| \leq 2$, we conclude, using (4.5) and Lebesgue's Theorem, that for any fixed $t \in\left[0, T^{*}\right)$ and $t_{n} \rightarrow t$

$$
\left|\theta^{\varepsilon_{n}, e_{n}}\left(\cdot, t_{n}\right)-\theta^{e}\left(\cdot, t_{n}\right)\right|_{\left.L^{1}\left(\mathbb{R}^{N}\right)\right)} \rightarrow 0 .
$$

Proceeding by contradiction, we see that this proof gives the existence of a modulus of continuity $\omega_{1}(\varepsilon)$ such that (4.4) holds. This ends the proof of the lemma. 


\section{Proof of Theorem 3.4}

We first recall that a function $\omega:[0,+\infty] \rightarrow[0,+\infty]$ is said to be a modulus of continuity, if $\omega$ is non decreasing and if $\omega(r) \rightarrow 0^{+}$as $r \rightarrow 0^{+}$. More generally, a function $\omega: \mathbb{R}^{d} \rightarrow[0,+\infty]$ is said to be a modulus of continuity if $r \mapsto \sup _{|\varepsilon| \leq r} \omega(\varepsilon)$ is a modulus of continuity.

Before to give the proof of Theorem 3.4, we need some preliminaries. First, we define

$$
\omega_{0}(r)= \begin{cases}C_{0} r^{\frac{1}{N}}+r & \text { if } \quad r \leq e_{0} \\ +\infty & \text { if } \quad r>e_{0}\end{cases}
$$

such that estimate (3.5) in assumption (A3) could be replaced by (for any $0<T \leq \min \left(\bar{T}, T^{*}-T_{0}\right)$ )

$$
\left|\theta^{e}-\theta\right|_{L^{\infty}\left(\left[0, T_{0}+T\right) ; L^{1}\left(\mathbb{R}^{N}\right)\right)} \leq C_{0}|e|_{L^{\infty}\left(\left[T_{0}, T_{0}+T\right)\right)} T+\omega_{0}\left(\left|\theta^{e}-\theta\right|_{L^{\infty}\left(\left[0, T_{0}\right] ; L^{1}\left(\mathbb{R}^{N}\right)\right)}\right) .
$$

We also have the following global in time estimate between $\theta^{e}$ and $\theta$ whose proof is postponed.

\section{Proposition 5.1 (Global estimate between $\theta^{e}$ and $\theta$ )}

Let $0<T \leq T^{*}$ and assume (A1)-(A2)-(A3). Then there exists a modulus of continuity $\tilde{\omega}$ (depending only on $T^{*}$ ) such that

$$
\left|\theta^{e}-\theta\right|_{L^{\infty}\left([0, T) ; L^{1}\left(\mathbb{R}^{N}\right)\right)} \leq \tilde{\omega}\left(|e|_{L^{\infty}([0, T))}\right) .
$$

To simplify the notation, we will set

$$
\left|c_{0}\right|_{\infty}=\left|c_{0}\right|_{L^{\infty}\left(\mathbb{R}^{N}\right)} .
$$

To prove Theorem 3.4, we need the following proposition.

\section{Proposition 5.2 (Iterative estimate)}

Under the assumptions of Theorem 3.4, up to decrease $\bar{T}$, we assume that $\bar{T} \leq \min \left(\frac{1}{4 C_{0}\left|c_{0}\right|_{\infty}}, 1\right)$ and that there exists a integer $K^{*} \geq 2$ such that $\frac{K^{*}}{2} \bar{T}=T^{*}$. We also assume that $\Delta t \leq \frac{\bar{T}}{6}$ and that there exists an integer $m \geq 0$ and a modulus of continuity $\bar{\omega}_{m}$ such that

$$
\begin{cases}\left|\theta^{\varepsilon}(\cdot, 0)-\theta(\cdot, 0)\right|_{\left.L^{1}\left(\mathbb{R}^{N}\right)\right)} \leq \bar{\omega}_{0}(\varepsilon) \quad \text { if } \quad m=0 \\ \left|\theta^{\varepsilon}-\theta\right|_{L^{\infty}\left(\left[0, \frac{m}{2} \bar{T}\right) ; L^{1}\left(\mathbb{R}^{N}\right)\right)} \leq \bar{\omega}_{m}(\varepsilon) \quad \text { if } \quad 1 \leq m \leq K^{*}-1\end{cases}
$$

Then there exists $\varepsilon_{m+1}>0$ such that we can define the following modulus of continuity

$$
\widehat{\omega}_{m+1}(\varepsilon)= \begin{cases}4\left(\omega_{1}(\varepsilon)+C_{0} \omega_{2}(\varepsilon)+\omega_{0}\left(\tilde{\omega}\left(\left|c_{0}\right|_{\infty} \bar{\omega}_{m}(\varepsilon)+\omega_{2}(\varepsilon)\right)\right)\right) & \text { if } \quad|\varepsilon| \leq \varepsilon_{m+1} \\ +\infty & \text { if } \quad|\varepsilon|>\varepsilon_{m+1}\end{cases}
$$

and

$$
\bar{\omega}_{m+1}(\varepsilon)=\max \left(\bar{\omega}_{m}(\varepsilon), \widehat{\omega}_{m+1}(\varepsilon)\right)
$$

where $\omega_{1}, \omega_{0}, \tilde{\omega}$ and $C_{0}$ are defined respectively in Proposition 4.5, in (5.1), in Proposition 5.1 and in assumption (A3), and $\omega_{2}(\varepsilon)=L_{c}(\Delta T+2 \Delta t)+L_{c_{1}} \Delta t$. Then we have

$$
\left|\theta^{\varepsilon}-\theta\right|_{L^{\infty}\left(\left[0, \frac{m+1}{2} \bar{T}\right) ; L^{1}\left(\mathbb{R}^{N}\right)\right)} \leq \bar{\omega}_{m+1}(\varepsilon) .
$$

\section{Proof of Theorem 3.4}

First remark that by our assumptions on the $C^{2}$ regularity of the bounded set $\Omega^{0}$, there exists a modulus of continuity $\bar{\omega}_{0}(\varepsilon)$ such that $(5.2)$ holds for $m=0$. Then we deduce from Proposition 5.2 that

$$
\left|\theta^{\varepsilon}-\theta\right|_{L^{\infty}\left(\left[0, T^{*}\right) ; L^{1}\left(\mathbb{R}^{N}\right)\right)} \leq \bar{\omega}_{K^{*}}(\varepsilon) .
$$

This ends the proof of the theorem. 
Before to do the proof of Proposition 5.2, let us explain the main idea, which is to use a barrier argument. To this aim, we have to introduce, for $e$ which will be chosen later, the function $\theta^{\varepsilon, e}$ (resp. $\theta^{\varepsilon,-e}$ ) given in (2.3) using the solution of the GFMM algorithm in Subsection 2.1 with a given local velocity $c[\theta(\cdot, t)](x, t)+e(t)$ (resp. $c[\theta(\cdot, t)](x, t)-e(t))$ where $\theta$ is the solution of (3.1) given in Assumption $(A 2)$. We also define $\theta^{e}$ (resp. $\left.\theta^{-e}\right)$ the exact solution of $(3.4)$ with speed $c[\theta(\cdot, t)](x, t)+e(t)(\operatorname{resp} . c[\theta(\cdot, t)](x, t)-e(t))$.

The idea is to choose $e$ such that

$$
\theta^{\varepsilon,-e} \leq \theta^{\varepsilon} \leq \theta^{\varepsilon, e}
$$

To show this result (see Lemma 5.3 below for a precise statement), we will use the comparison principle of the monotone GFMM (Theorem 4.1). To estimate the difference between $\theta$ and $\theta^{\varepsilon}$, we will then use the fact that $\theta^{\varepsilon, \pm e}$ is closed to $\theta^{ \pm e}$ (by the convergence result of the monotone GFMM, see Lemma 4.5) and the fact that $\theta^{ \pm e}$ is closed to $\theta$ (by Assumption (A3)).

For simplicity of notation we define $c(x, t)=c[\theta(\cdot, t)](x, t)$. We also need to define the velocity $c^{\varepsilon}$ that is used in the non-local GFMM (see Step 10b of the non-local GFMM). To do this, we define iteratively $c^{\varepsilon}\left(x, t_{n_{k}}\right)$, where $\left(t_{n_{k}}\right)_{k}$ is the increasing sequence defined in (2.2) for the non-local GFMM given in Subsection 2.2 , by setting the initial velocity

$$
c^{\varepsilon}(x, 0) \equiv c\left[\theta^{\varepsilon}(\cdot, 0)\right](x, 0)
$$

and if $t_{n_{k-1}} \in[p \Delta T,(p+1) \Delta T)$ for some $p \in \mathbb{N}$, then

$$
c^{\varepsilon}\left(x, t_{n_{k}}\right)= \begin{cases}c^{\varepsilon}\left(x, t_{n_{k-1}}\right) & \text { if } \quad t_{n_{k}}<(p+1) \Delta T \\ c\left[\theta^{\varepsilon}\left(\cdot, t_{n_{k-1}}\right)\right]\left(x, t_{n_{k}}\right) & \text { if } \quad t_{n_{k}} \geq(p+1) \Delta T\end{cases}
$$

We extend the velocity in time by considering

$$
c^{\varepsilon}(x, t)=c^{\varepsilon}\left(x, t_{n_{k}}\right) \quad \text { for } t \in\left[t_{n_{k}}, t_{n_{k+1}}\right) .
$$

Using the definition (2.3) of $\theta^{\varepsilon}$, we see that the function $\theta^{\varepsilon}$ is also given by the (local) GFMM algorithm with the new velocity $c^{\varepsilon}$.

Before to give the proof of Proposition 5.2, we need the following lemma whose proof is postponed.

\section{Lemma 5.3 (Barriers on $\theta^{\varepsilon}$ )}

Let $\tilde{e}:[0,+\infty) \mapsto[0,+\infty)$ be a non-decreasing function.

We assume that

$$
\tilde{e}(0) \geq\left|\left(\theta^{\varepsilon}-\theta\right)(\cdot, 0)\right|_{L^{1}\left(\mathbb{R}^{N}\right)}
$$

Moreover if $t_{n_{k}}>0$, we assume that

$$
\forall t_{n_{l}} \in\left(0, t_{n_{k}}\right), \quad \tilde{e}\left(\max \left(t_{n_{l}}-\Delta t, 0\right)\right) \geq\left|\theta^{\varepsilon}-\theta\right|_{L^{\infty}\left(\left[0, t_{n_{l}}\right) ; L^{1}\left(\mathbb{R}^{N}\right)\right)}
$$

We define

$$
e(t)=\left|c_{0}\right|_{\infty} \tilde{e}(t)+\omega_{2}(\varepsilon) \quad \text { with } \omega_{2}(\varepsilon)=L_{c}(\Delta T+2 \Delta t)+L_{c_{1}} \Delta t .
$$

Then for all $(x, t) \in \mathbb{R}^{N} \times\left[0, t_{n_{k+1}}\right)$, we have

$$
\theta^{\varepsilon,-e}(x, t) \leq \theta^{\varepsilon}(x, t) \leq \theta^{\varepsilon, e}(x, t) .
$$

\section{Proof of Proposition 5.2}

Let us denote by $\left(t_{n_{k}}\right)_{k}$ the sequence of increasing time corresponding to the non-local GFMM for $\theta^{\varepsilon}$ defined in (2.2). For any integer $m$ satisfying $0 \leq m \leq K^{*}-1$, we define $p$ and $k$ such that

$$
0 \leq t_{n_{k}} \leq \frac{m}{2} \bar{T}<t_{n_{k+1}}<\cdots<t_{n_{k+p}} \leq \frac{m+1}{2} \bar{T}<t_{n_{k+p+1}}
$$

In particular, we have $t_{n_{k+p+1}} \in\left(\frac{m+1}{2} \bar{T}, \frac{m+1}{2} \bar{T}+\Delta t\right]$.

We proceed by recurrence. We assume that there exists $l \in\{0, \ldots, p\}$ such that

$$
\left|\theta^{\varepsilon}-\theta\right|_{L^{\infty}\left(\left[0, t_{n_{k+l}}\right) ; L^{1}\left(\mathbb{R}^{N}\right)\right)} \leq \bar{\omega}_{m+1}(\varepsilon) \text { if } \quad t_{n_{k+l}}>0
$$


We exclude the case $m=l=0$, because we recall that $t_{n_{0}}=0=t_{0}$. Remark also that inequality (5.6) is always satisfied for $l=0$ when $m \geq 1$, because of our assumption (5.2), the fact that $t_{n_{k}} \leq \frac{m}{2} \bar{T}$ and the fact that by definition of $\bar{\omega}_{m+1}(\varepsilon)$, we have $\bar{\omega}_{m}(\varepsilon) \leq \bar{\omega}_{m+1}(\varepsilon)$.

Then we will show that

$$
\left|\theta^{\varepsilon}-\theta\right|_{L^{\infty}\left(\left[0, t_{n_{k+l+1}}\right) ; L^{1}\left(\mathbb{R}^{N}\right)\right)} \leq \bar{\omega}_{m+1}(\varepsilon)
$$

where in the special case where $m=K^{*}-1$, we redefine $t_{n_{k+p+1}}$ to be equal to $\frac{K^{*}}{2} \bar{T}=T^{*}$, in order to insure that for any integer $0 \leq m \leq K^{*}-1$, we have $t_{n_{k+p+1}} \leq T^{*}$.

We define $\varepsilon_{m+1}$ such that for all $|\varepsilon| \leq \varepsilon_{m+1}$, we have

$$
\left\{\begin{array}{l}
\bar{\omega}_{m+1}(\varepsilon) \leq \frac{e_{0} \bar{T}}{\left.\left.6\right|_{0}\right|_{\infty}} \\
\left|c_{0}\right|_{\infty} \bar{\omega}_{m+1}(\varepsilon)+\omega_{2}(\varepsilon) \leq e_{0} \\
\tilde{\omega}\left(\left|c_{0}\right|_{\infty} \bar{\omega}_{m}(\varepsilon)+\omega_{2}(\varepsilon)\right) \leq e_{0}
\end{array}\right.
$$

The proof is decomposed into two cases.

Case 1: $m \geq 1$.

In that case, remark that $t_{n_{k}}>0$ (otherwise $t_{n_{k+1}} \leq \Delta t \leq \frac{\bar{T}}{6}<\frac{m}{2} \bar{T}$ ). We claim that for $|\varepsilon| \leq \varepsilon_{m+1}$, | $\theta^{\varepsilon}-$ $\left.\theta\right|_{L^{\infty}\left(\left[0, t_{n_{k+l+1}}\right) ; L^{1}\left(\mathbb{R}^{N}\right)\right)}$ satisfies (5.7). To show this, let us define a non-decreasing function $\tilde{e} \in W^{1, \infty}([0, \infty))$ such that in the case $l=0$

$$
\tilde{e}(t)=\left|\theta^{\varepsilon}-\theta\right|_{L^{\infty}\left(\left[0, t_{n_{k}}\right) ; L^{1}\left(\mathbb{R}^{N}\right)\right)} \quad \text { if } \quad t \geq 0
$$

and we set in the case $l \geq 1$

$$
\tilde{e}(t)= \begin{cases}\left|\theta^{\varepsilon}-\theta\right|_{L^{\infty}\left(\left[0, \frac{m}{2} \bar{T}\right) ; L^{1}\left(\mathbb{R}^{N}\right)\right)} & \text { if } \quad t \leq\left(\frac{m}{2}-\frac{1}{3}\right) \bar{T} \\ \left|\theta^{\varepsilon}-\theta\right|_{L^{\infty}\left(\left[0, t_{n_{k+l}}\right) ; L^{1}\left(\mathbb{R}^{N}\right)\right)} & \text { if } \quad t \geq\left(\frac{m}{2}-\frac{1}{6}\right) \bar{T}\end{cases}
$$

with $\tilde{e}$ linear on $\left[\left(\frac{m}{2}-\frac{1}{3}\right) \bar{T},\left(\frac{m}{2}-\frac{1}{6}\right) \bar{T}\right]$. We also define $e(t)=\left|c_{0}\right|_{\infty} \tilde{e}(t)+\omega_{2}(\varepsilon)$. Using estimate (5.8), we easily check that $e \in \mathcal{E}$ (where $\mathcal{E}$ is defined in (4.3) with $e_{0}$ given in assumption (A3)).

Using Lemma 5.3 and the fact that $t_{n_{k+l+1}} \leq \min \left(\left(\frac{m}{2}+\frac{2}{3}\right) \bar{T}, T^{*}\right)$, we then get

$$
\begin{aligned}
\left|\left(\theta^{\varepsilon}-\theta\right)^{+}\right|_{L^{\infty}\left(\left[0, t_{\left.\left.n_{k+l+1}\right) ; L^{1}\left(\mathbb{R}^{N}\right)\right)}\right.\right.} & \leq\left|\left(\theta^{\varepsilon, e}-\theta\right)^{+}\right|_{L^{\infty}\left(\left[0, t_{n_{k+l+1}}\right) ; L^{1}\left(\mathbb{R}^{N}\right)\right)} \\
& \leq\left|\left(\theta^{\varepsilon, e}-\theta^{e}\right)^{+}\right|_{L^{\infty}\left(\left[0, T^{*}\right) ; L^{1}\left(\mathbb{R}^{N}\right)\right)}+\left|\left(\theta^{e}-\theta\right)^{+}\right|_{L^{\infty}\left(\left[0, \min \left(\left(\frac{m}{2}+\frac{2}{3}\right) \bar{T}, T^{*}\right)\right) ; L^{1}\left(\mathbb{R}^{N}\right)\right)} \\
& \leq \omega_{1}(\varepsilon)+C_{0}|e|_{L^{\infty}([0, \infty))} \bar{T}+\omega_{0}\left(\left|\theta^{e}-\theta\right|_{L^{\infty}\left(\left[0,\left(\frac{m}{2}-\frac{1}{3}\right) \bar{T}\right] ; L^{1}\left(\mathbb{R}^{N}\right)\right)}\right)
\end{aligned}
$$

where, in the last line, we have used Proposition 4.5 to bound the first term and estimate (5.1) (implied by Assumption $(A 3))$ to bound the second term.

In the same way we obtain

$$
\left|\left(\theta^{\varepsilon}-\theta\right)^{-}\right|_{L^{\infty}\left(\left[0, t_{n_{k+l+1}}\right) ; L^{1}\left(\mathbb{R}^{N}\right)\right)} \leq \omega_{1}(\varepsilon)+C_{0}|e|_{L^{\infty}([0, \infty))} \bar{T}+\omega_{0}\left(\left|\theta^{e}-\theta\right|_{L^{\infty}\left(\left[0,\left(\frac{m}{2}-\frac{1}{3}\right) \bar{T}\right] ; L^{1}\left(\mathbb{R}^{N}\right)\right)}\right)
$$

Summing (5.9) and (5.10), we get

$$
\begin{aligned}
\left|\theta^{\varepsilon}-\theta\right|_{L^{\infty}\left(\left[0, t_{n_{k+l+1}}\right) ; L^{1}\left(\mathbb{R}^{N}\right)\right) \leq} & 2 C_{0}\left(\left|c_{0}\right|_{\infty}\left|\theta^{\varepsilon}-\theta\right|_{L^{\infty}\left(\left[0, t_{n_{k+l}}\right) ; L^{1}\left(\mathbb{R}^{N}\right)\right)}+\omega_{2}(\varepsilon)\right) \bar{T} \\
& +2 \omega_{1}(\varepsilon)+2 \omega_{0}\left(\left|\theta^{e}-\theta\right|_{L^{\infty}\left(\left[0,\left(\frac{m}{2}-\frac{1}{3}\right) \bar{T}\right] ; L^{1}\left(\mathbb{R}^{N}\right)\right)}\right) \\
\leq & \frac{1}{2}\left|\theta^{\varepsilon}-\theta\right|_{L^{\infty}\left(\left[0, t_{n_{k+l}}\right) ; L^{1}\left(\mathbb{R}^{N}\right)\right)}+2\left(C_{0} \omega_{2}(\varepsilon)+\omega_{1}(\varepsilon)\right. \\
& \left.\quad+\omega_{0}\left(\tilde{\omega}\left(\left|c_{0}\right|_{\infty}\left|\theta^{\varepsilon}-\theta\right|_{L^{\infty}\left(\left[0, \frac{m}{2} \bar{T}\right) ; L^{1}\left(\mathbb{R}^{N}\right)\right)}+\omega_{2}(\varepsilon)\right)\right)\right) \\
\leq & \frac{1}{2}\left|\theta^{\varepsilon}-\theta\right|_{L^{\infty}\left(\left[0, t_{n_{k+l}}\right) ; L^{1}\left(\mathbb{R}^{N}\right)\right)}+\frac{1}{2} \bar{\omega}_{m+1}(\varepsilon) \\
\leq & \bar{\omega}_{m+1}(\varepsilon)
\end{aligned}
$$


where we have used the fact that $\bar{T} \leq \min \left(\frac{1}{4 C_{0}\left|c_{0}\right|_{\infty}}, 1\right)$ and Proposition 5.1 for the second inequality, the fact that $\left|\theta^{\varepsilon}-\theta\right|_{L^{\infty}\left(\left[0, \frac{m}{2} \bar{T}\right) ; L^{1}\left(\mathbb{R}^{N}\right)\right)} \leq \bar{\omega}_{m}(\varepsilon)$ joint to the definition of $\bar{\omega}_{m+1}$ for the third one and (5.6) for the last one. This proves (5.7)

Case 2: $m=0$.

The proof is completely similar to the previous one. The only difference is that we take the constant function $\tilde{e}=\left|\theta^{\varepsilon}-\theta\right|_{L^{\infty}\left(\left[0, t_{n_{k+l}}\right) ; L^{1}\left(\mathbb{R}^{N}\right)\right)}$ (or the constant function $\tilde{e}=\left|\theta^{\varepsilon}(\cdot, 0)-\theta(\cdot, 0)\right|_{L^{1}\left(\mathbb{R}^{N}\right)}$ if $t_{n_{k+l}}=0$ ) and that (5.9) is replaced by

$$
\left|\left(\theta^{\varepsilon}-\theta\right)^{+}\right|_{L^{\infty}\left(\left[0, t_{n_{k+l+1}}\right) ; L^{1}\left(\mathbb{R}^{N}\right)\right)} \leq \omega_{1}(\varepsilon)+C_{0} e \bar{T}+\omega_{0}\left(\left|\theta^{e}(\cdot, 0)-\theta(\cdot, 0)\right|_{L^{1}\left(\mathbb{R}^{N}\right)}\right)=\omega_{1}(\varepsilon)+C_{0} e \bar{T} .
$$

Hence, we will get

$$
\begin{aligned}
\left|\theta^{\varepsilon}-\theta\right|_{L^{\infty}\left(\left[0, t_{n_{k+l+1}}\right) ; L^{1}\left(\mathbb{R}^{N}\right)\right)} & \leq 2 C_{0}\left(\left|c_{0}\right|_{\infty} \bar{\omega}_{1}(\varepsilon)+\omega_{2}(\varepsilon)\right) \bar{T}+2 \omega_{1}(\varepsilon) \\
& \leq \frac{1}{2} \bar{\omega}_{1}(\varepsilon)+2\left(C_{0} \omega_{2}(\varepsilon)+\omega_{1}(\varepsilon)\right) \\
& \leq \bar{\omega}_{1}(\varepsilon)
\end{aligned}
$$

This proves (5.7) and ends the proof of the theorem.

\section{Proof of Lemma 5.3}

Let $x \in \mathbb{R}^{N}$ and $0 \leq t_{n_{l}} \leq t_{n_{k}}$. We define $p$ such that $t_{n_{l}} \in[p \Delta T,(p+1) \Delta T)$.

We claim that

$$
c^{\varepsilon}\left(x, t_{n_{l}}\right) \leq \inf _{s \in\left[\max \left(t_{n_{l}}-\Delta t, 0\right), \min \left(t_{n_{l}}+\Delta t, t_{n_{k+1}}\right)\right]} c^{e}(x, s) .
$$

In the case $p=0$, we extend $\theta^{\varepsilon}$ for negative times as

$$
\theta^{\varepsilon}(x, t)=\theta_{I}^{0} \quad \text { if } \quad(x, t) \in\left[x_{I}, x_{I}+\Delta x\right) \times(-\infty, 0)
$$

Then, by definition of $c^{\varepsilon}$, there exists two times $t^{*} \in[p \Delta T-\Delta t, p \Delta T)$ and $t^{* *} \in\left[t^{*}, t^{*}+\Delta t\right]$ with $t^{* *} \geq p \Delta T$ such that

$$
c^{\varepsilon}\left(x, t_{n_{l}}\right)=c_{0} \star \theta^{\varepsilon}\left(\cdot, t^{*}\right)(x)+c_{1}\left(x, t^{* *}\right) .
$$

We define $\bar{s}$ such that $\inf _{s \in\left[\max \left(t_{n_{l}}-\Delta t, 0\right), \min \left(t_{n_{l}}+\Delta t, t_{n_{k+1}}\right)\right]}(c(x, s)+e(s))=c(x, \bar{s})+e(\bar{s})$, then we have

$$
\begin{aligned}
c^{\varepsilon}\left(x, t_{n_{l}}\right)= & c_{0} \star\left(\theta^{\varepsilon}\left(\cdot, t^{*}\right)-\theta\left(\cdot, t^{*}\right)\right)(x)+c\left[\theta\left(\cdot, t^{*}\right)\right]\left(x, t^{*}\right) \\
& +c_{1}\left(x, t^{* *}\right)-c_{1}\left(x, t^{*}\right)-c[\theta(\cdot, \bar{s})](x, \bar{s})+c[\theta(\cdot, \bar{s})](x, \bar{s}) \\
\leq & \left|c_{0}\right|_{\infty}\left|\theta^{\varepsilon}\left(\cdot, t^{*}\right)-\theta\left(\cdot, t^{*}\right)\right|_{L^{1}\left(\mathbb{R}^{N}\right)}+L_{c}\left|t^{*}-\bar{s}\right| \\
& +L_{c_{1}}\left|t^{*}-t^{* *}\right|+c[\theta(\cdot, \bar{s})](x, \bar{s}) \\
\leq & \left|c_{0}\right|_{\infty} \tilde{e}\left(\max \left(t^{*}-\Delta t, 0\right)\right)+L_{c}(\Delta T+2 \Delta t)+L_{c_{1}} \Delta t+c(x, \bar{s}) \\
\leq & \left|c_{0}\right|_{\infty} \tilde{e}(\bar{s})+L_{c}(\Delta T+2 \Delta t)+L_{c_{1}} \Delta t+c(x, \bar{s}) \\
= & e(\bar{s})+c(x, \bar{s})=\inf _{s \in\left[\max \left(t_{n_{l}}-\Delta t, 0\right), \min \left(t_{n_{l}}+\Delta t, t_{n_{k+1}}\right)\right]}(c(x, s)+e(s))
\end{aligned}
$$

where we have used for the fourth inequality the fact $\max \left(t^{*}-\Delta t, 0\right) \leq \max \left(t_{n_{l}}-\Delta t, 0\right) \leq \bar{s}$ and the monotonicity of the function $\tilde{e}$, and for the third inequality the fact that $0 \leq \bar{s}-t^{*} \leq\left|t^{*}-p \Delta T\right|+\mid p \Delta T-$ $t_{n_{l}}|+| t_{n_{l}}-\bar{s} \mid \leq \Delta T+2 \Delta t$, that $\left|t^{*}-t^{* *}\right| \leq \Delta t$ and that $t^{*}<t_{n_{l}} \leq t_{n_{k}}$ to apply (5.4). This proves the desired result (5.11). 
We can then apply the comparison principle Theorem 4.1 to the couple $\left(\theta^{\varepsilon, e}, \theta^{\varepsilon}\right)$, and we get for all $(x, t) \in \mathbb{R}^{N} \times\left[0, t_{n_{k+1}}\right):$

$$
\theta^{\varepsilon}(x, t) \leq \theta^{\varepsilon, e}(x, t)
$$

Similarly, we prove that

$$
c^{\varepsilon}\left(x, t_{n_{l}}\right) \geq \sup _{s \in\left[\max (t-\Delta t, 0), \min \left(t+\Delta t, t_{n_{k}}\right)\right]} c^{-e}(x, s)
$$

and then that $\theta^{\varepsilon,-e} \leq \theta^{\varepsilon}$.

This ends the proof of the lemma.

We finish this section by the proof of Proposition 5.1.

\section{Proof of Proposition 5.1}

Up to decrease $\bar{T}$, we assume that there exists an integer $K \geq 3$ such that $\frac{K}{2} \bar{T}=T$ with $\bar{T} \leq 1$ (remark that $K$ can be bounded by a constant depending only on $T^{*}$ and $\bar{T}$ ).

We argue by recurrence. First using (A3), we get that

$$
\begin{aligned}
\left|\theta^{e}-\theta\right|_{L^{\infty}\left([0, \bar{T}) ; L^{1}\left(\mathbb{R}^{N}\right)\right)} & \leq C_{0}|e|_{L^{\infty}([0, \bar{T}))} \bar{T} \\
& \leq C_{0}|e|_{L^{\infty}([0, T))}=: \tilde{\omega}_{2}\left(|e|_{L^{\infty}([0, T))}\right) .
\end{aligned}
$$

where we have used the fact that $\bar{T} \leq 1$.

Now, let us assume that there exists $k \in\{2, \ldots, K-1\}$ and a modulus of continuity $\tilde{\omega}_{k}$ such that

$$
\left|\theta^{e}-\theta\right|_{L^{\infty}\left(\left[0, \frac{k}{2} \bar{T}\right) ; L^{1}\left(\mathbb{R}^{N}\right)\right)} \leq \tilde{\omega}_{k}\left(|e|_{L^{\infty}([0, T))}\right)
$$

and we will prove that

$$
\left|\theta^{e}-\theta\right|_{L^{\infty}\left(\left[0, \frac{k+1}{2} \bar{T}\right) ; L^{1}\left(\mathbb{R}^{N}\right)\right)} \leq \tilde{\omega}_{k+1}\left(|e|_{L^{\infty}([0, T))}\right)
$$

where

$$
\tilde{\omega}_{k+1}(r)= \begin{cases}C_{0} r+\omega_{0}\left(\tilde{\omega}_{k}(r)\right) & \text { if } r \leq e_{k+1} \\ +\infty & \text { if } r>e_{k+1} .\end{cases}
$$

for some constant $e_{k+1} \leq e_{0}$ chosen such that

$$
r \leq e_{k+1} \quad \Longrightarrow \quad \tilde{\omega}_{k}(r) \leq e_{0}
$$

The results will then follow taking

$$
\tilde{\omega}(r):=\omega_{K}(r)
$$

To show (5.12), we use assumption (A3) and (5.1), and we get for $2 \leq k \leq K-1$ that

$$
\begin{aligned}
\left|\theta^{e}-\theta\right|_{L^{\infty}\left(\left[0, \frac{k+1}{2} \bar{T}\right) ; L^{1}\left(\mathbb{R}^{N}\right)\right)} & \leq C_{0}|e|_{L^{\infty}([0, T))} \bar{T}+\omega_{0}\left(\left|\theta^{e}-\theta\right|_{L^{\infty}\left(\left[0, \frac{k-1}{2} \bar{T}\right] ; L^{1}\left(\mathbb{R}^{N}\right)\right)}\right) \\
& \leq C_{0}|e|_{L^{\infty}([0, T))} \bar{T}+\omega_{0}\left(\left|\theta^{e}-\theta\right|_{L^{\infty}\left(\left[0, \frac{k}{2} \bar{T}\right) ; L^{1}\left(\mathbb{R}^{N}\right)\right)}\right) \\
& \leq \tilde{\omega}_{k+1}\left(|e|_{L^{\infty}([0, T))}\right) .
\end{aligned}
$$

where we have used the fact that $\bar{T} \leq 1$ to get the last line.

This proves (5.12) and ends the proof of the proposition. 


\section{Proof of Theorem 3.1: short time evolution with non signed velocity}

We consider the case of a closed dislocation loop which is a smooth curve $\partial \Omega_{0}$ in $\mathbb{R}^{2}$ at the initial time. For short time evolution, it is known that the curve stays Lipschitz (see [4]). In this subsection, we prove that under an additional regularity assumption on $\partial \Omega_{0}$, (A1) implies (A2)-(A3).

\section{Lemma 6.1 (Checking assumption (A2))}

Assume (A1) and that $\Omega_{0} \subset \mathbb{R}^{2}$ is a $C^{3}$ bounded connected and simply connected open set. Then there exists $T_{1}^{*}>0$ (depending only on $\Omega_{0}$ and on bounds on $\left|c_{1}\right|_{L^{\infty}\left(\mathbb{R}^{2} \times[0,+\infty)\right)}+\left|\nabla c_{1}\right|_{L^{\infty}\left(\mathbb{R}^{2} \times[0,+\infty)\right)}$ and on $\left.\left|c_{0}\right|_{L^{\infty}\left(\mathbb{R}^{2}\right)}+\left|c_{0}\right|_{W^{1,1}\left(\mathbb{R}^{2}\right)}\right)$ for which the following holds:

\section{i) (Existence and uniqueness)}

There exists a unique function $\theta \in C\left(\left[0, T_{1}^{*}\right) ; L^{1}\left(\mathbb{R}^{2}\right)\right)$ which is a discontinuous viscosity solution of equation (3.1).

The function $\theta$ can be written as

$$
\theta(\cdot, t)=1_{\Omega_{f(\cdot, t)}}-1_{\Omega_{f(, t)}^{c}}
$$

where $\Omega_{f(\cdot, t)}$ is an open set of boundary $\Gamma_{f}$ where $\Gamma_{f(\cdot, t)}(s)=\Gamma(s)+f(s, t) n(s)$ with $\Gamma(s)$ a parametrization of $\partial \Omega_{0}$ with unit normal $n(s)$. Here the function $f$ satisfies the equation:

$$
\left\{\begin{array}{lll}
f_{t}(s, t)=\left(c_{1}-\int_{\mathbb{R}^{2}} c_{0}+2 c_{0} \star 1_{\Omega_{f(\cdot, t)}}\right)\left(\Gamma_{f(\cdot, t)}(s), t\right) \sqrt{1+\left(\left(\frac{1}{1-f K(s)}\right) f_{s}\right)^{2}} & \text { on } & \mathbb{R} \times\left(0, T_{1}^{*}\right) \\
f(s+L, t)=f(s, t) & \text { on } & \mathbb{R} \times\left(0, T_{1}^{*}\right) \\
f(\cdot, 0)=0 & \text { on } \mathbb{R}
\end{array}\right.
$$

where $f_{s}=\frac{\partial f}{\partial s}, K(s)$ is the curvature of $\partial \Omega_{0}$ at the point $\Gamma(s)$ and $L$ is the length of $\partial \Omega_{0}$. Moreover

$$
|f|_{L^{\infty}\left(\mathbb{R} \times\left[0, T_{1}^{*}\right)\right)} \leq \frac{1}{2|K|_{L^{\infty}(\mathbb{R})}}
$$

and there exists a constant $L_{1}>0$ (depending only on $\Omega_{0}$ and on the bounds on $c_{1}$ and $c_{0}$ ) such that

$$
|f|_{W^{1, \infty}\left(\mathbb{R} \times\left[0, T_{1}^{*}\right)\right)} \leq L_{1}
$$

ii) (Velocity Lipschitz in time)

The speed $c[\theta(\cdot, t)]$ satisfies $(3.3)$.

Before to prove Lemma 6.1, let us write the following result whose the proof is postponed (and which will be used in the proofs in the sequel):

\section{Lemma 6.2 ( $L^{\infty}$ versus $L^{1}$ estimate)}

We use the notation of Lemma 6.1.

i) $L^{\infty}-L^{1}$ estimate

There exists a constant $C_{0}>0$ such that for any functions $f^{1}, f^{2}$ satisfying

$$
\left|f^{i}\right|_{L^{\infty}(\mathbb{R})} \leq \frac{1}{2|K|_{L^{\infty}(\mathbb{R})}} \quad \text { for } \quad i=1,2
$$

we have

$$
\left|1_{\Omega_{f^{2}}}-1_{\Omega_{f^{1}}}\right|_{L^{1}\left(\mathbb{R}^{2}\right)} \leq C_{0}\left|f^{2}-f^{1}\right|_{L^{\infty}(\mathbb{R})}
$$

ii) $L^{1}-L^{\infty}$ estimate

Given a constant $L_{1}>0$, there exists a constant $C_{1}>0$ such that if we have

$$
\left|f_{s}^{i}\right|_{L^{\infty}(\mathbb{R})} \leq L_{1} \quad \text { and } \quad\left|f^{i}\right|_{L^{\infty}(\mathbb{R})} \leq \frac{1}{2|K|_{L^{\infty}(\mathbb{R})}} \quad \text { for } \quad i=1,2
$$

then

$$
\left|f^{2}-f^{1}\right|_{L^{\infty}(\mathbb{R})} \leq C_{1}\left|1_{\Omega_{f^{2}}}-1_{\Omega_{f^{1}}}\right|_{L^{1}(\mathbb{R})}^{\frac{1}{2}}
$$




\section{Proof of Lemma 6.1}

\section{Proof of point i)}

Let us define $\bar{c}_{0}(x)=\sup _{y \in B_{1}(y)}\left|c_{0}(y)\right|$. Under the additional assumption

$$
\bar{c}_{0} \in L^{1}\left(\mathbb{R}^{2}\right)
$$

and

$$
c_{1} \in W^{1, \infty}\left(\mathbb{R}^{2} \times[0,+\infty)\right)
$$

then point i) follows from the statement of Theorem 1.3 in [4] (and estimate (6.2) follows from the proofs of Theorem 3.7 and Theorem 4.3 of [4]). Assumption (6.4) was introduced in [4] to be able to deal with unbounded open set $\Omega_{0}$. But looking at the proof of Theorem 1.3 in [4] (see in particular the proof of Theorem 4.3), we see that the result is still true without assumption (6.4) (for a bounded open set $\Omega_{0}$ ).

Looking more carefully at the proof of [4], we see that we only use the weaker condition

$$
c_{1} \in C\left(\mathbb{R}^{2} \times[0,+\infty)\right) \text { and } \nabla c_{1} \in L^{\infty}\left(\mathbb{R}^{2} \times[0,+\infty)\right) .
$$

This shows point i) for some $T_{1}^{*}>0$.

Proof of point ii)

Using moreover Corollary 4.4 of [4], we see that $f \in W^{1, \infty}\left(\mathbb{R} \times\left(0, T_{1}^{*}\right)\right)$, and then we have with $\theta(x, t)=$ $21_{\Omega_{f(, t)}}(x)-1$ and $s>0$

$$
\begin{aligned}
|c[\theta(\cdot, t+s)](x, t+s)-c[\theta(\cdot, t)](x, t)| & \leq\left|c_{1}(x, t+s)-c_{1}(x, t)\right|+2\left|c_{0}\right|_{L^{\infty}\left(\mathbb{R}^{2}\right)}\left|1_{\Omega_{f(\cdot, t+s)}}-1_{\Omega_{f(\cdot, t)}}\right|_{L^{1}\left(\mathbb{R}^{2}\right)} \\
& \leq L_{c} s
\end{aligned}
$$

with

$$
L_{c}=\left|\left(c_{1}\right)_{t}\right|_{L^{\infty}\left(\mathbb{R}^{2} \times\left(0, T_{1}^{*}\right)\right)}+2\left|c_{0}\right|_{L^{\infty}\left(\mathbb{R}^{2}\right)} C_{0} L_{1}
$$

where $L_{1}$ is given in (6.2) and we have used estimate (6.3). This shows that $c[\theta(\cdot, t)]$ satisfies $(3.3)$ and ends the proof of point ii).

\section{Proof of Lemma 6.2}

\section{Proof of i)}

Estimate (6.3) is simple to prove (see estimate (4.44) in the proof of Theorem 4.3 in [4]).

\section{Proof of ii)}

Let $\delta=\left|f^{2}-f^{1}\right|_{L^{\infty}(\mathbb{R})}$. Up to exchange the role of $f^{2}$ and $f^{1}$, we can assume that $\delta=f^{2}\left(s_{0}\right)-f^{1}\left(s_{0}\right)$ for some point $s_{0} \in \mathbb{R}$. Then for any $|h| \leq h^{*}:=\delta /\left(2 L_{1}\right)$, we have

$$
f^{2}\left(s_{0}+h\right) \geq f^{2}\left(s_{0}\right)-L_{1}|h| \geq f^{1}\left(s_{0}\right)+L_{1}|h| \geq f^{1}\left(s_{0}+h\right)
$$

Then it is natural to define the set

$$
E=\left\{(r, h), \quad h \in\left(-h^{*}, h^{*}\right), r \in\left(f^{1}\left(s_{0}\right)+L_{1}|h|, f^{2}\left(s_{0}\right)-L_{1}|h|\right)\right\}
$$

and the map $\Psi(r, h):=\Gamma\left(s_{0}+h\right)+r n\left(s_{0}+h\right)$. Therefore, we see that by assumption we have

$$
\Psi(E) \subset \Omega_{f^{2}} \backslash \Omega_{f^{1}}
$$

On the other hand the set $E$ is a losange of diagonals of length $2 h^{*}$ and $\delta$, which implies

$$
|E|=\delta h^{*} .
$$

Therefore, we have with $C_{2}=\inf _{E}|\operatorname{Jac} \Psi|$ :

$$
|\Psi(E)| \geq C_{2}|E|=C_{2} \delta h^{*}=\left(\delta / C_{1}\right)^{2}
$$

which defines the constant $C_{1}$. Then the result follows from (6.6). This ends the proof of the lemma. 
Lemma 6.3 (Checking assumption (A3))

Given $\Omega_{0}$ as in Lemma 6.1 and a velocity $c \in W^{1, \infty}\left(\mathbb{R}^{2} \times\left[0, T_{1}^{*}\right)\right)$, for any function $e$, we consider the velocity

$$
c^{e}(x, t)=c(x, t)+e(t)
$$

Then there exists $e_{0}>0$ and $T^{*} \in\left(0, T_{1}^{*}\right]$ such that for any $e \in C\left(\left[0, T^{*}\right)\right)$ satisfying $|e|_{L^{\infty}\left(\left[0, T^{*}\right)\right)} \leq e_{0}$, there exists a unique viscosity solution $\theta^{e}$ of the local equation

$$
\begin{cases}\theta_{t}^{e}(x, t)=c^{e}(x, t)\left|D \theta^{e}(x, t)\right| & \text { in } \mathbb{R}^{2} \times\left(0, T^{*}\right) \\ \theta^{e}(\cdot, 0)=1_{\Omega_{0}}-1_{\Omega_{0}^{c}} & \text { on } \mathbb{R}^{2}\end{cases}
$$

Moreover

$$
\left|\left(\theta^{e}\right)^{*}(\cdot, t)-\left(\theta^{e}\right)_{*}(\cdot, t)\right|_{L^{1}\left(\mathbb{R}^{2}\right)}=0 \quad \text { for all } t \in\left[0, T^{*}\right)
$$

and there exists constants $C_{0}>0$ and $\bar{T}>0$ such that for every $0 \leq T_{0}<T^{*}$, if $\left|\left(\theta^{e}-\theta\right)\left(\cdot, T_{0}\right)\right|_{L^{1}\left(\mathbb{R}^{2}\right)} \leq e_{0}$, then we have

$$
\left|\theta^{e}-\theta\right|_{L^{\infty}\left(\left[T_{0}, T_{0}+T\right) ; L^{1}\left(\mathbb{R}^{2}\right)\right)} \leq C_{0}\left(|e|_{L^{\infty}\left(\left[T_{0}, T_{0}+T\right)\right)} T+\left|\left(\theta^{e}-\theta\right)\left(\cdot, T_{0}\right)\right|_{L^{1}\left(\mathbb{R}^{2}\right)}^{\frac{1}{2}}\right)
$$

with $0<T \leq \min \left(\bar{T}, T^{*}-T_{0}\right)$.

\section{Proof of Lemma 6.3}

\section{Step 1: Existence and uniqueness}

We simply apply Lemma 6.1 in the case $c_{0}=0$, i.e. a local equation, and $c_{1}=c^{e}$. This gives the existence and uniqueness of a solution on a time interval $\left[0, T^{*}\right)$ with $T^{*} \in\left(0, T_{1}^{*}\right]$ which only depends on $\Omega_{0}$ and on bounds on $\left|c^{e}\right|_{L^{\infty}\left(\mathbb{R}^{2} \times\left[0, T^{*}\right)\right)}+\left|\nabla c^{e}\right|_{L^{\infty}\left(\mathbb{R}^{2} \times\left[0, T^{*}\right)\right)}$, i.e. on bounds on $|c|_{W^{1, \infty}\left(\mathbb{R}^{2} \times\left[0, T^{*}\right)\right)}$ and on $e_{0} \geq|e|_{L^{\infty}\left(\left[0, T^{*}\right)\right)}$. Then (6.8) follows from the fact that

$$
\theta^{e}=1_{\Omega_{f}(\cdot, t)}-1_{\Omega_{f e(\cdot, t)}^{c}}
$$

Here the function $f^{e}(s, t)$ is solution of

$$
\left\{\begin{array}{lll}
f_{t}^{e}=H^{e}\left(s, t, f^{e}, f_{s}^{e}\right) & \text { on } & \mathbb{R} \times\left(0, T^{*}\right) \\
f^{e}(s+L, t)=f^{e}(s, t) & \text { on } & \mathbb{R} \times\left(0, T^{*}\right) \\
f^{e}(\cdot, 0)=0 & \text { on } & \mathbb{R}
\end{array}\right.
$$

with

$$
H^{e}(s, t, f, p)=c^{e}\left(\Gamma_{f}(s), t\right) \sqrt{1+\left(\frac{p}{1-f K(s)}\right)^{2}}
$$

and

$$
\Gamma_{f}(s)=\Gamma(s)+f n(s)
$$

where $\Gamma(s), n(s), K(s)$ and $f$ are defined in Lemma 6.1. Moreover, up to redefine the constant $L_{1}$, we have

$$
\left|f^{e}\right|_{W^{1, \infty}\left(\mathbb{R} \times\left[0, T^{*}\right)\right)} \leq L_{1}
$$

for all $|e|_{L^{\infty}\left(\left[0, T^{*}\right)\right)} \leq e_{0}$.

Step 2: Bound on $\left|f^{e}-f\right|_{L^{\infty}\left(\mathbb{R} \times\left[T_{0}, T_{0}+T\right)\right)}$ for any $0 \leq T_{0}<T^{*}$ and $0<T \leq \min \left(\bar{T}, T^{*}-T_{0}\right)$ Let us define

$$
\hat{f}^{e}(s, t)=f(s, t)+C \bar{e}\left(t-T_{0}\right)+a \quad \text { with } \quad \bar{e}=|e|_{L^{\infty}\left(\left[T_{0}, T_{0}+T\right)\right.}+a \quad \text { and } \quad a=\left|f^{e}\left(\cdot, T_{0}\right)-f\left(\cdot, T_{0}\right)\right|_{L^{\infty}(\mathbb{R})}
$$

For $\left|f_{2}\right|,\left|f_{1}\right| \leq \delta_{0}:=\frac{1}{2|K|_{L^{\infty}(\mathbb{R})}}$, we have with $H:=H^{0}$

$$
\left|H\left(s, t, f_{2}, p\right)-H\left(s, t, f_{1}, p\right)\right| \leq L_{2}(1+|p|)\left|f_{2}-f_{1}\right|
$$

for some constant $L_{2}$ (see for instance in [4], both the end of the proof of theorem 4.3 and inequality (3.27)). Up to reduce respectively $T^{*}$ and $e_{0}$, we can assume that

$$
|f|_{L^{\infty}\left(\mathbb{R} \times\left[0, T^{*}\right)\right)} \leq \delta_{0} / 3 \text { and } e_{0} \leq \delta_{0} / 3
$$


Finally, assuming that

$$
a \leq e_{0} \quad \text { and } \quad 2 e_{0} C \bar{T} \leq \delta_{0} / 3
$$

we compute for $T^{*} \geq T_{0}+T>t \geq T_{0}$

$$
\begin{aligned}
\hat{f}_{t}^{e} & \geq H\left(s, t, f, f_{s}\right)+C \bar{e} \\
& \geq H\left(s, t, \hat{f}^{e}, f_{s}\right)+C \bar{e}-L_{2}\left(1+L_{1}\right)\left(C \bar{e}\left(t-T_{0}\right)+a\right) \\
& \geq H^{e}\left(s, t, \hat{f}^{e}, f_{s}\right)-|e|_{L^{\infty}\left(\left[T_{0}, T_{0}+T\right)\right)}\left(1+2 L_{1}\right)+C \bar{e}\left(1-\left(t-T_{0}\right) L_{2}\left(1+L_{1}\right)\right)-a L_{2}\left(1+L_{1}\right) \\
& \geq H^{e}\left(s, t, \hat{f}^{e}, \hat{f}_{s}^{e}\right)+C \bar{e} / 4
\end{aligned}
$$

where we have used (6.11) for the second line, (6.12) and the fact that $|e|_{L^{\infty}\left(\left[0, T^{*}\right)\right)} \leq e_{0}$ for the third line, and we have assumed $\bar{T} \leq\left(2 L_{2}\left(1+L_{1}\right)\right)^{-1}$ and $C>0$ large enough for the last line $\left(C \geq 4 \max \left(1+2 L_{1}, L_{2}(1+\right.\right.$ $\left.\left.L_{1}\right)\right)$ ). This is a classical exercise to check that we have in the viscosity sense

$$
\hat{f}_{t}^{e} \geq H^{e}\left(s, t, \hat{f}^{e}, \hat{f}_{s}^{e}\right) \quad \text { on } \quad \mathbb{R} \times\left(T_{0}, T_{0}+T\right)
$$

Then from the comparison principle, we deduce that

$$
f^{e} \leq \hat{f}^{e} \quad \text { on } \quad \mathbb{R} \times\left[T_{0}, T_{0}+T\right)
$$

Similarly, we can prove that

$$
f^{e} \geq \hat{f}^{-e} \quad \text { with } \quad \hat{f}^{-e}(s, t)=f(s, t)-C \bar{e}\left(t-T_{0}\right)-a
$$

which implies for $|e|_{L^{\infty}\left(\left[0, T^{*}\right)\right)} \leq e_{0}$ :

$$
\left|f^{e}-f\right|_{L^{\infty}\left(\mathbb{R} \times\left[T_{0}, T_{0}+T\right)\right)} \leq C \bar{e} T+a \quad \text { for any } \quad 0 \leq T_{0}<T_{0}+T \leq T^{*} \quad \text { with } \quad 0<T \leq \bar{T}
$$

\section{Step 3: Conclusion}

Estimate (6.13) joint to (6.3) and Lemma 6.2 ii) implies estimate (6.9) with another constant $C>0$.

\section{Proof of Theorem 3.1}

By Lemmata 6.1 and 6.3, assumptions (A2)-(A3) are verified, and then the result follows from Theorem 3.4.

\section{Proof of Theorem 3.2: large time evolution with positive veloc- ity}

In this section, we consider the case of dislocation dynamics with positive velocity. In this case, under a strengthened assumption (A1), we prove that (A2) and (A3) hold.

\section{Lemma 7.1 (Checking assumption (A2))}

Assume that $c_{0} \in W^{2,1}\left(\mathbb{R}^{N}\right) \cap L^{\infty}\left(\mathbb{R}^{N}\right), c_{1} \in C\left(\mathbb{R}^{N} \times[0,+\infty)\right)$ with $\nabla c_{1}, D^{2} c_{1} \in L^{\infty}\left(\mathbb{R}^{N} \times[0,+\infty)\right)$ and $c_{1}(x, t) \geq\left|c_{0}\right|_{L^{1}}$. Assume also that $\Omega_{0} \subset \mathbb{R}^{N}$ is a bounded $C^{2}$ open set. Then the following holds:

(Existence and uniqueness) There exists a unique discontinuous viscosity solution $\theta \in C\left([0, \infty) ; L^{1}\left(\mathbb{R}^{N}\right)\right)$ of equation (3.1). Moreover, we have

$$
\left|\theta^{*}(\cdot, t)-\theta_{*}(\cdot, t)\right|_{L^{1}\left(\mathbb{R}^{2}\right)}=0 \quad \text { for all } \quad t \in[0,+\infty)
$$

(Velocity Lipschitz in time) The speed $c[\theta(\cdot, t)]$ satisfies (3.3). 


\section{Proof of Lemma 7.1}

If we assume that the velocity have higher local regularity, namely $c_{0} \in W^{2, \infty}\left(\mathbb{R}^{N}\right) \cap L^{1}\left(\mathbb{R}^{N}\right), c_{1} \in$ $W^{2, \infty}\left(\mathbb{R}^{N} \times[0,+\infty)\right)$, then the existence and uniqueness is given by [1, Theorem 4.3]. Equality (7.1) follows from [1, Lemma 3.1]. The Lipschitz in time property $(3.3)$ of $c[\theta(\cdot, t)](x, t)$ comes from [1, Proposition 3.3] applied to the case with two velocities $\tilde{c}_{1}(x, s)=c[\theta(\cdot, s+t)](x, s+t)$ and $\tilde{c}_{2}=0$ with initial condition $\theta(\cdot, t)$ joint to the following inequality

$$
\left|c\left[\theta^{1}(\cdot, t)\right](\cdot, t)-c\left[\theta^{2}(\cdot, t)\right](\cdot, t)\right|_{L^{\infty}\left(\mathbb{R}^{N}\right)} \leq\left|c_{0}\right|_{\infty}\left|\theta^{1}(\cdot, t)-\theta^{2}(\cdot, t)\right|_{\left.L^{1}\left(\mathbb{R}^{N}\right)\right)} .
$$

From the proofs of the previous mentioned results, it is possible to see that the same result holds when we weaken the assumptions on $c_{0}, c_{1}$ as in the statement of Lemma 7.1 (see in particular Theorem 1.3, case ii) of [5] where this result is true for the level set formulation of the problem).

\section{Definition 7.2 (Interior ball property)}

We say that a compact set $K$ of $\mathbb{R}^{N}$ has the interior ball property of radius $r>0$, if and only if for any $y \in \partial K$, there exists a ball $B_{r}(x) \subset K$ such that $y \in \partial B_{r}(x)$.

\section{Definition 7.3 (Hausdorff distance)}

Let $K^{1}$ and $K^{2}$ two compact sets of $\mathbb{R}^{N}$. Then we define the Hausdorff distance between $K^{1}$ and $K^{2}$ as

$$
\operatorname{dist}_{\mathcal{H}}\left(K^{1}, K^{2}\right)=\max \left(\sup _{x \in K^{1}} \inf _{y \in K^{2}}|x-y|, \sup _{x \in K^{2}} \inf _{y \in K^{1}}|x-y|\right) .
$$

\section{Proposition 7.4 (Hausdorff distance controlled by the Lebesgue measure)}

Let the radii $R>r>0$ be fixed. Then there exists a constant $C=C(R, r, N)>0$ such that the following holds. Let $K^{1}$ and $K^{2}$ be two compact sets contained in the ball $B_{R}(0)$ of $\mathbb{R}^{N}$. Assume that $K^{1}$ and $K^{2}$ have the interior ball property of radius $r$. Then we have

$$
\operatorname{dist}_{\mathcal{H}}\left(K^{1}, K^{2}\right) \leq C\left|K^{1} \Delta K^{2}\right|^{\frac{1}{N}}
$$

where $|A|$ is the Lebesgue measure of a set $A$, and $K^{1} \Delta K^{2}=\left(K^{1} \backslash K^{2}\right) \cup\left(K^{2} \backslash K^{1}\right)$.

\section{Proof of Proposition 7.4}

Without loss of generality, let us assume that

$$
\rho=\operatorname{dist}_{\mathcal{H}}\left(K^{1}, K^{2}\right)=\sup _{x \in K^{2}} \operatorname{dist}\left(x, K^{1}\right)=\operatorname{dist}\left(x_{0}, K^{1}\right),
$$

with $x_{0} \in K^{2}$. This implies in particular that

$$
B_{\rho}\left(x_{0}\right) \cap K^{1}=\emptyset .
$$

The proof is decomposed into two cases:

Case 1: there exists $x_{1} \in\left(\partial K^{2}\right) \cap B_{\rho / 2}\left(x_{0}\right)$. This implies that

$$
B_{\rho / 2}\left(x_{1}\right) \cap K^{1}=\emptyset .
$$

Using the fact that $K^{2}$ satisfies the interior ball property of radius $r$, we deduce that there exists $z_{1} \in K^{2}$ such that

$$
B_{r}\left(z_{1}\right) \subset K^{2} \text { and } \quad x_{1} \in \partial B_{r}\left(z_{1}\right) .
$$

Sub-case 1.1: $\frac{\rho}{2} \leq r$. In this case, we deduce that

$$
B_{\rho / 2}\left(z_{0}\right) \subset B_{r}\left(z_{1}\right)
$$

where $z_{0}=x_{1}+\frac{\rho}{2} \frac{z_{1}-x_{1}}{\left|z_{1}-x_{1}\right|}$. Hence

$$
B_{\rho / 2}\left(z_{0}\right) \cap B_{\rho / 2}\left(x_{1}\right) \subset B_{r}\left(z_{1}\right) \cap B_{\rho / 2}\left(x_{1}\right) \subset K^{2} \backslash K^{1} \subset K^{1} \Delta K^{2} .
$$


This implies that there exists a constant $C_{2}$ (depending only on $N$ ) such that

$$
\left|K^{1} \Delta K^{2}\right| \geq C_{2} \rho^{N}
$$

and so

$$
\operatorname{dist}_{\mathcal{H}}\left(K^{1}, K^{2}\right)=\rho \leq\left(\frac{\left|K^{1} \Delta K^{2}\right|}{C_{2}}\right)^{\frac{1}{N}}
$$

Sub-case 1.2: $\frac{\rho}{2} \geq r$. In this case, we deduce that

$$
B_{r}\left(z_{1}\right) \cap B_{r}\left(x_{1}\right) \subset K^{2} \backslash K^{1} \subset K^{1} \Delta K^{2} .
$$

This implies that that there exists a constant $C_{3}$ (depending only on $N$ ) such that

$$
\left|K^{1} \Delta K^{2}\right| \geq C_{3} r^{N}
$$

and so

$$
\operatorname{dist}_{\mathcal{H}}\left(K^{1}, K^{2}\right) \leq 2 R \leq \frac{2 R}{r}\left(\frac{\left|K^{1} \Delta K^{2}\right|}{C_{3}}\right)^{\frac{1}{N}}
$$

Case 2: $B_{\rho / 2}\left(x_{0}\right) \subset K^{2} \backslash K^{1}$. In this case, there exists a constant $C_{4}$ such that

$$
\left|K^{1} \Delta K^{2}\right| \geq C_{4} \rho^{N}
$$

and so

$$
\operatorname{dist}_{\mathcal{H}}\left(K^{1}, K^{2}\right)=\rho \leq\left(\frac{\left|K^{1} \Delta K^{2}\right|}{C_{4}}\right)^{\frac{1}{N}} .
$$

Finally in the general case, we see that (7.3) holds with a constant $C$ only depending on the radii $R, r$ and the dimension $N$.

\section{Lemma 7.5 (Checking assumption (A3))}

Assume that $\Omega_{0} \subset \mathbb{R}^{N}$ is a bounded $C^{2}$ open set and let $c \in C\left(\mathbb{R}^{N} \times[0, \infty)\right)$ with $\nabla c, D^{2} c \in L^{\infty}\left(\mathbb{R}^{N} \times[0,+\infty)\right)$, satisfying $c \geq \delta>0$, and for any $e \in C([0, \infty))$ with $|e|_{L^{\infty}([0, \infty))}<\delta$, let us set

$$
c^{e}(x, t)=c(x, t)+e(t)
$$

Then there exists a unique viscosity solution $\theta^{e}$ of the local equation

$$
\left\{\begin{array}{lll}
\theta_{t}^{e}(x, t)=c^{e}(x, t)\left|D \theta^{e}(x, t)\right| & \text { in } & \mathbb{R}^{N} \times(0,+\infty) \\
\theta^{e}(\cdot, 0)=1_{\Omega_{0}}-1_{\Omega_{0}^{c}} & \text { on } & \mathbb{R}^{N}
\end{array}\right.
$$

and

$$
\left|\left(\theta^{e}\right)^{*}(\cdot, t)-\left(\theta^{e}\right)_{*}(\cdot, t)\right|_{L^{1}\left(\mathbb{R}^{N}\right)}=0 \quad \text { for all } \quad t \in[0,+\infty)
$$

Moreover for every $T^{*}>0$, there exists $e_{0} \in(0, \delta), C_{0}>0$ and $\bar{T}>0$ such that for any $0 \leq T_{0}<T^{*}$, and if the following holds

$$
|e|_{L^{\infty}\left(\left[0, T^{*}\right)\right)} \leq e_{0} \quad \text { and } \quad\left|\theta^{e}-\theta\right|_{L^{\infty}\left(\left[0, T^{*}\right) ; L^{1}\left(\mathbb{R}^{N}\right)\right)} \leq e_{0}
$$

then we have

$$
\left|\theta^{e}-\theta\right|_{L^{\infty}\left(\left[T_{0}, T_{0}+T\right) ; L^{1}\left(\mathbb{R}^{N}\right)\right)} \leq C_{0}\left(|e|_{L^{\infty}\left(\left[T_{0}, T_{0}+T\right)\right)} T+\left|\left(\theta^{e}-\theta\right)\left(\cdot, T_{0}\right)\right|_{L^{1}\left(\mathbb{R}^{N}\right)}^{\frac{1}{N}}\right)
$$

for $0<T \leq \min \left(\bar{T}, T^{*}-T_{0}\right)$. 


\section{Proof of Lemma 7.5}

Because $c \geq \delta$, and $|e|_{L^{\infty}([0,+\infty))}<\delta$, Lemma 7.1 applies and proves the existence and uniqueness of a solution satisfying (7.5). Moreover, for any $T^{*}>0$, applying [1, Propositions 3.3] to equation (7.4) with speeds $c$ and $c^{e}$, this gives the existence of $e_{0}, C_{1}>0$ such that

$$
\left|\theta^{e}-\theta\right|_{L^{\infty}\left(\left[T_{0}, T_{0}+T\right) ; L^{1}\left(\mathbb{R}^{N}\right)\right)} \leq C_{1}\left(|e|_{L^{\infty}\left(\left[T_{0}, T_{0}+T\right)\right)} T+\operatorname{dist}_{\mathcal{H}}\left(\left\{\theta^{e}\left(\cdot, T_{0}\right)=1\right\},\left\{\theta\left(\cdot, T_{0}\right)=1\right\}\right)\right)
$$

for $T_{0}<T_{0}+T \leq T^{*}$ with $T \leq \bar{T}$ for some $\bar{T}>0$. Now using Proposition 7.4 joint to the estimate on the radius $r=r\left(T_{0}\right)$ of the interior ball given in [1, Lemma 3.1], we deduce that

$$
\operatorname{dist}_{\mathcal{H}}\left(\left\{\theta^{e}\left(\cdot, T_{0}\right)=1\right\},\left\{\theta\left(\cdot, T_{0}\right)=1\right\}\right) \leq C_{2}\left|\theta^{e}\left(\cdot, T_{0}\right)-\theta\left(\cdot, T_{0}\right)\right|_{L^{1}\left(\mathbb{R}^{N}\right)}^{\frac{1}{N}}
$$

Finally (7.7) and (7.8) imply (7.6).

\section{Proof of Theorem 3.2}

By Lemmata 7.1 and 7.5, assumptions (A2), (A3) are verified, and then the result follows from Theorem 3.4 .

\section{Numerical tests for dislocation dynamics}

We present some numerical tests performed with a slightly modified non-local GFMM (see Appendix A). These tests simulate dislocation dynamics in dimension $N=2$.

The non-local part of the speed is given by $c_{0} \star \theta^{+}$, where $\theta^{+}=\frac{\theta+1}{2}$ is the positive part of $\theta$. This problem is equivalent to (3.1), up to rescaling the kernel $c_{0}$ and modifying the local speed $c_{1}$. In the sequel, for simplicity we will denote $\theta^{+}$by $\theta$.

To compute the non-local discrete velocity $c^{\varepsilon}(x, t)=c\left[\theta^{\varepsilon}(\cdot, t)\right](x, t)+c_{1}(x, t)$, we have to compute a convolution of the form

$$
\left(c_{0} \star \theta^{\varepsilon}\left(\cdot, t_{n_{k}}\right)\right)\left(x_{I}\right) \text {. }
$$

This convolution can be rewritten in the following sum:

$$
\left(c_{0} \star \theta^{\varepsilon}\left(\cdot, t_{n_{k}}\right)\left(x_{I}\right)=\left(c_{0} \star\left(\theta^{n_{k+1}-1}\right)^{\Delta}\left(x_{I}\right)=\sum_{J \in \mathbb{Z}^{2}} \bar{c}_{0, I-J} \theta_{J}^{n_{k+1}-1},\right.\right.
$$

where $(\theta)^{\Delta}$ has been defined in (2.4) and

$$
\bar{c}_{0, I}=\frac{1}{\left|Q_{I}\right|} \int_{Q_{I}} c_{0}(x) d x
$$

with $Q_{I}$ the square cell centred in $x_{I}$ :

$$
Q_{I}=\left[x_{i_{1}}-\Delta x / 2, x_{i_{1}}+\Delta x / 2\right] \times\left[x_{i_{2}}-\Delta x / 2, x_{i_{2}}+\Delta x / 2\right] .
$$

The kernel $c_{0}=c_{0, \delta}$ is chosen as the kernel proposed in [4] for the Peierls-Nabarro model, which Fourier transform is given by:

$$
\widehat{c}_{0, \delta}\left(\xi_{x_{1}}, \xi_{x_{2}}\right)=-\frac{1}{2}\left(\frac{\xi_{x_{1}}^{2}+\left(\frac{1}{1-\nu}\right) \xi_{x_{2}}^{2}}{\sqrt{\xi_{x_{1}}^{2}+\xi_{x_{2}}^{2}}}\right) e^{-\delta \sqrt{\xi_{x_{1}}^{2}+\xi_{x_{2}}^{2}}}
$$

where $\nu$ is the Poisson ratio that takes values in $(-1,0.5)$, and $\delta$ is a constant proportional to the size of the core of the dislocations. In our simulations we consider cases with $\delta=C \Delta x$, where $1 \leq C \leq 11$. Since $c_{0}$ tends to 0 for $|x| \rightarrow \infty$, we truncate $c_{0, \delta}$ outside the numerical domain.

We consider a bounded square $D=[-l, l] \times[-l, l]$ as numerical domain. In the case $\theta\left(x_{1}, x_{2}\right)$ has a compact support contained in the numerical domain (Test 2), we assume $\theta\left(x_{1}, x_{2}\right) 2 l$-periodic in both directions. Then the discrete convolution is computed on $D$ by the inverse discrete Fourier transform of the product of the Fourier transform of $c_{0}$ with the Fourier transform of $\theta\left(x_{1}, x_{2}\right)$ (for more details about the computation of the convolution we refer to [3]). 
In other cases (Test 1 and Test 3 ) we choose an initial data which is $2 l$-periodic in the $x_{2}-$ direction. Then the discrete convolution (8.1) is calculated on a larger numerical domain $D_{K}=[-(2 K+1) l,(2 K+1) l] \times$ $[-(2 K+1) l,(2 K+1) l]$, where $K$ is a positive integer. The kernel $c^{0}$ is now truncated outside $D_{K}$ and the following extension of $\theta$ is used in the computation of the speed. First, $\theta\left(x_{1}, x_{2}\right)$ is extended over the domain $[-(2 K+1) l,-l] \times[-l, l]$ by the constant in $x_{2}$ value $\theta(-l, \cdot)$ and on the domain $[l,(2 K+1) l] \times[-l, l]$ by the constant in $x_{2}$ value $\theta(l, \cdot)$. Then it is extended over all the domain $D_{K}$ periodically in the $x_{2}-$ direction.

The non local speed is computed each time step size $\Delta T$, whose value will be specified in each test. The step $\Delta t$ is always chosen equal to the space step $\Delta x$.

Test 1 : a propagating line with obstacles

We choose as initial data a line and three different speeds describing three different obstacles. In all cases the continuous speed is given by $c(x, t)=\left(c_{0, \delta} \star \theta(\cdot, t)\right)(x)+c_{1}(x, t)$ with $\delta=0.5, \nu=0$ and local speed

$$
c_{1}(x, t)=\left\{\begin{array}{ll}
\alpha & \text { if }|x|^{2}<0.3 \\
2 & \text { otherwise }
\end{array} .\right.
$$

The presence of the external force $c_{1}$ allows the line to propagate. For $\alpha<2$, the speed $c_{1}$ on the domain $|x|^{2}<0.3$ can be thought as an obstacle which slows down the propagation of the line. Smaller $\alpha$ is, deeper the obstacle is. We compute the discrete solution in the numerical domain $[-3,3] \times[-3,3]$, with 129 nodes each sides and we update the speed each $\Delta T=0.02$. The discrete convolution is computed in the extended domain $D_{K}$ with $K=1$ and $l=3$.

Case $\alpha=1.5$ : the line passes over the obstacle

The line comes slowed down in the zone of the obstacle, but it succeeds to exceed it without to disconnect itself. In Figure 2 we show the line evolution and the obstacle at times $t=0,0.42,0.66,0.93,1.03,1.44$.

Case $\alpha=1$ : the obstacle breaks the line

Lowering the value of $\alpha$ but holding it still positive, we simulate the presence of a deeper obstacle that slows down the propagation of the line. Due to the presence of this deeper obstacle, the line disconnects. Therefore there is a change of topology. One part of the dislocation still propagates to the left, the other part remains captured by the obstacle and collapses over time. In Figure 3 we show the line evolution and the obstacle at times $t=0,0.38,0.69,0.85,1.05,1.20$.

Case $\alpha=-0.5$ : the obstacle captures the line

Choosing a deeper obstacle, the line does not succeed to exceed the obstacle. There is a change of topology: a part exceeds the obstacle and continues propagating to the left, another part remains captured around to the obstacle. In Figure 4, we show the line evolution and the obstacle at times $t=0,0.47,0.74,1.04,1.35,1.81$.

\section{Test 2 : Shrinking of a square}

Here, we are looking at the collapse of a non smooth front: the square.

We choose as continuous speed $\left.c(x, t)=\left(c_{0, \delta} \star \theta\right)(\cdot, t)\right)(x)$ with $\delta=0.02$ and $\nu=0$. In this case the shrinking of the square approaches a circle, this is expected since for $\delta \rightarrow 0$ the dislocation dynamics approaches the Mean Curvature Motion.

We compute the discrete solution in the numerical domain $[-3,3] \times[-3,3]$, with 160 nodes each sides and we update the speed each $\Delta T=0.005$. We plot the contour line each 0.025 time step.

This test has been done in a precedent paper with a finite difference scheme for the level set formulation, see $[3]$.

Since for very small core $\delta$, the speed has large gradient, the finite difference scheme was not satisfactory: the gradient of the solution becomes too small and it is hard to follow the level set evolution.

We compare the numerical solution given by the GFMM algorithm (Subsection 2.2) with the one given by the Level Sets method with a finite difference scheme.

The finite difference scheme produces oscillations in the level set line representing the dislocation and it is not possible to follow the complete evolution (see Figure 5) on the left. This fact happens because the continuous function, which 0 contour line represents the fronts, has became flat. 

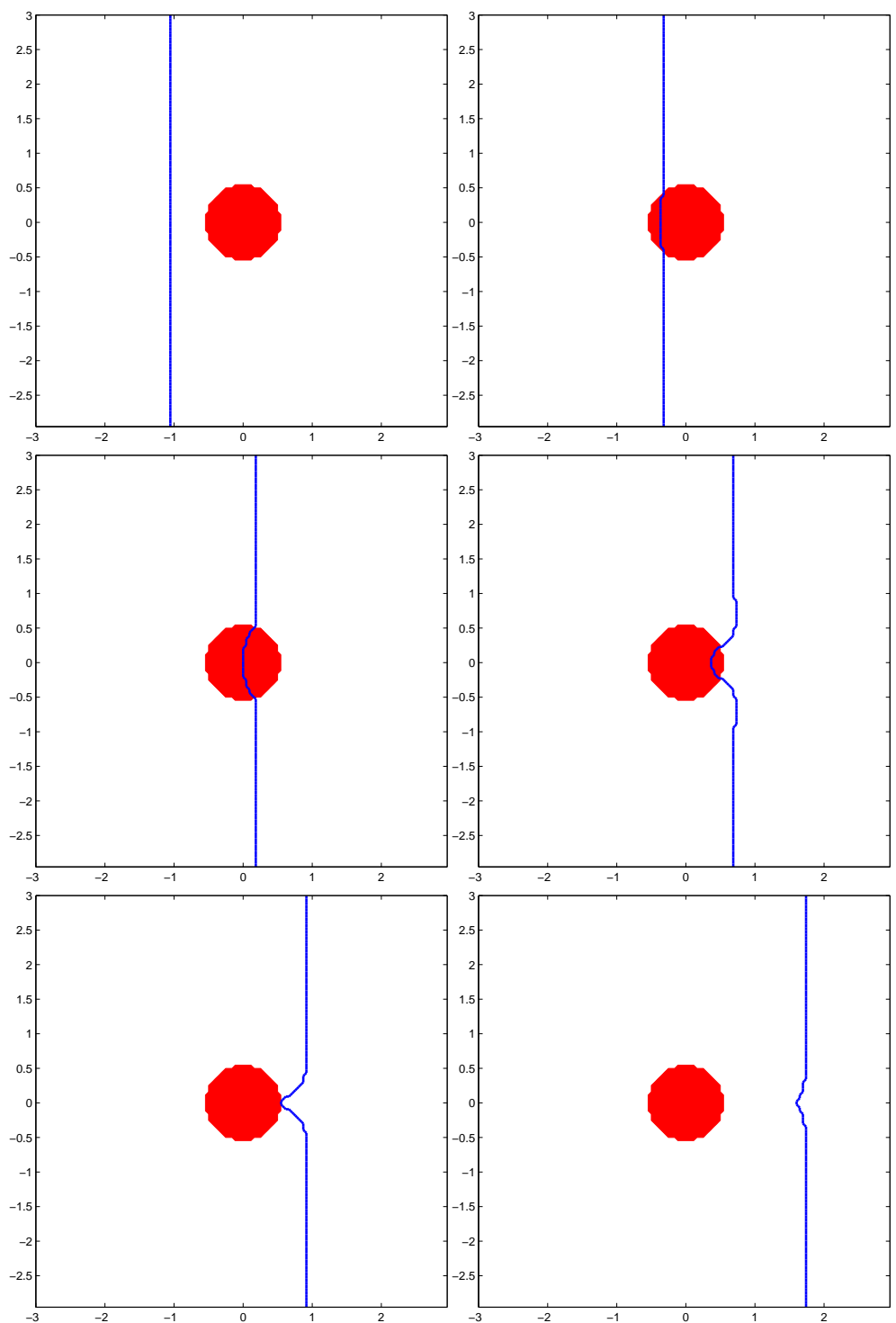

Figure 2: A propagating line with an obstacle, case $\alpha=1.5$

The GFMM does not suffer of this problem: no oscillations are produced in the evolution and it is possible to follow the evolution until the square collapse, see Figure 5 on the right.

\section{Test 3 : Collapse of a sinusoidal line dislocation}

We choose as initial data a sinusoidal line $x_{1}=-0.15 \sin \left(\pi x_{2}\right)$ and as speed $\left.c(x, t)=\left(c_{0, \delta} \star \theta\right)(\cdot, t)\right)(x)$ with $\delta=0.005$ and $\nu=0$. In this case the speed changes sign with respect to the space variable. It is expected that a sinusoidal line will collapse to a straight line.

We compute the discrete solution in the numerical domain $[-1,1] \times[-1,1]$, with 100 nodes each side. The discrete convolution is computed in the extended domain $D_{K}$ with $K=4$ and it is updated each $\Delta T=10^{-4}$. In Fig. 6 we show the numerical solution at times $t=0,2.15 \cdot 10^{-3}, 4.06 \cdot 10^{-3}, 4.89 \cdot 10^{-3}, 6.57 \cdot 10^{-3}, 1.19 \cdot 10^{-2}$. 


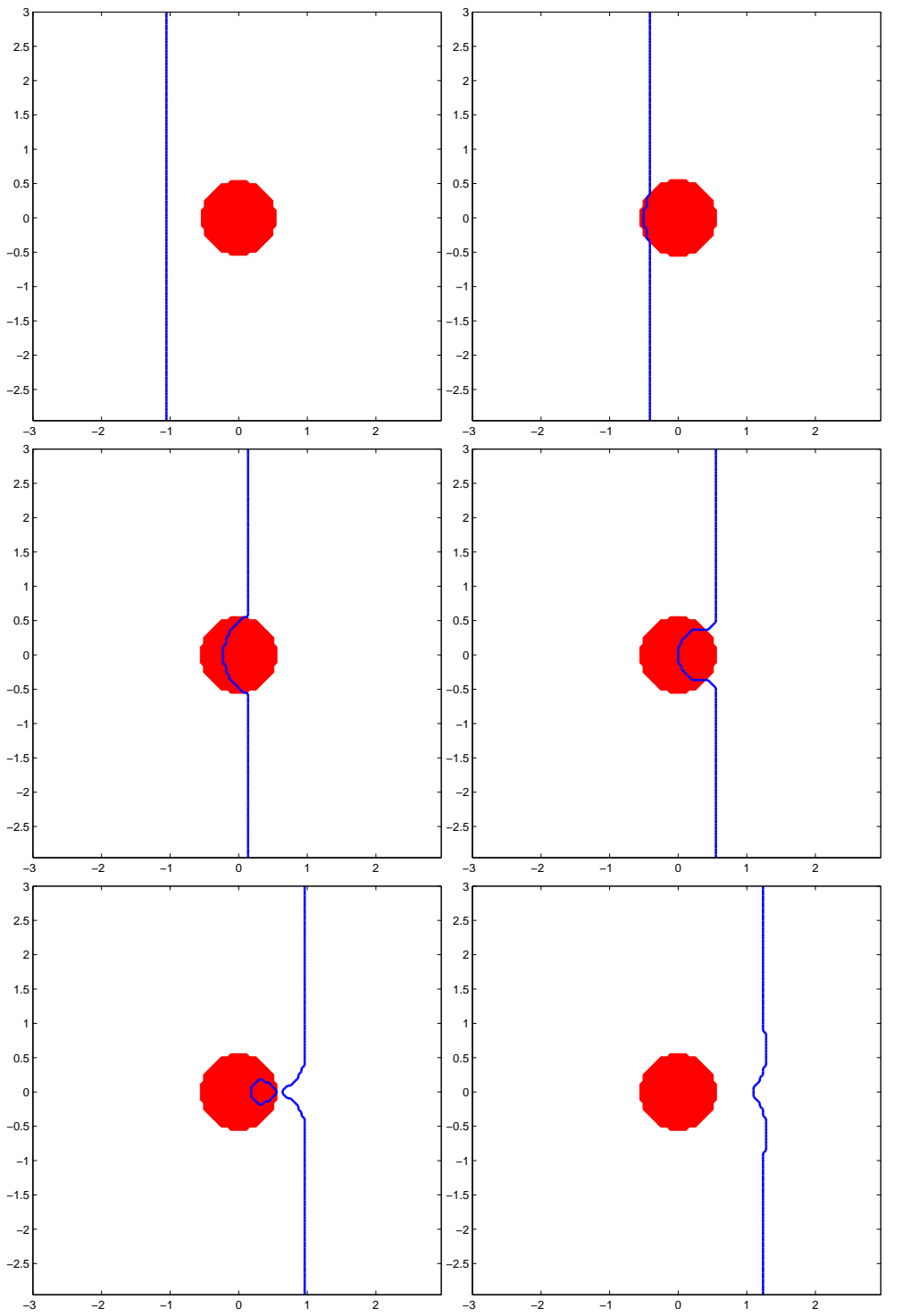

Figure 3: A propagating line with an obstacle, case $\alpha=1$ 


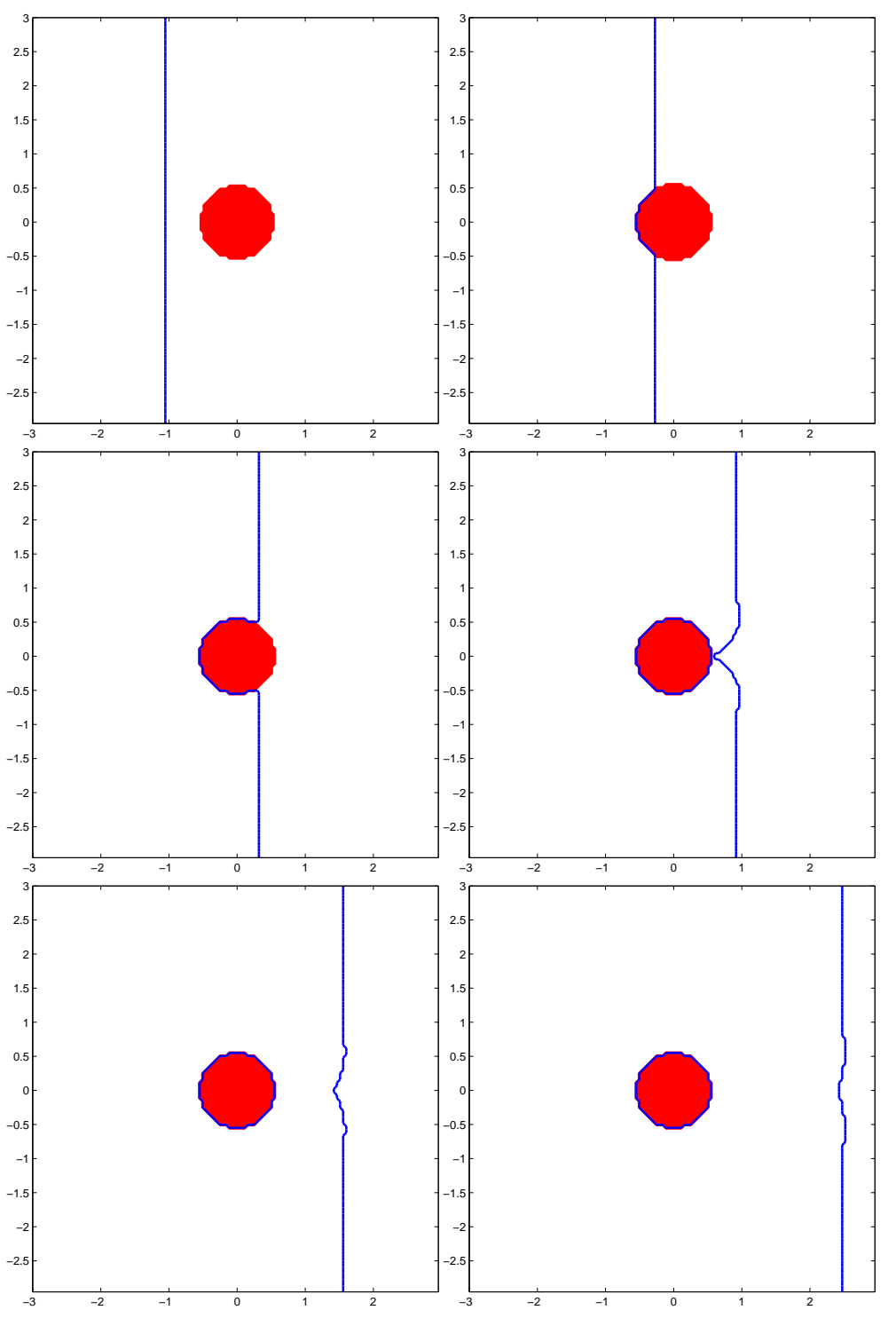

Figure 4: A propagating line with an obstacle, case $\alpha=-0.5$
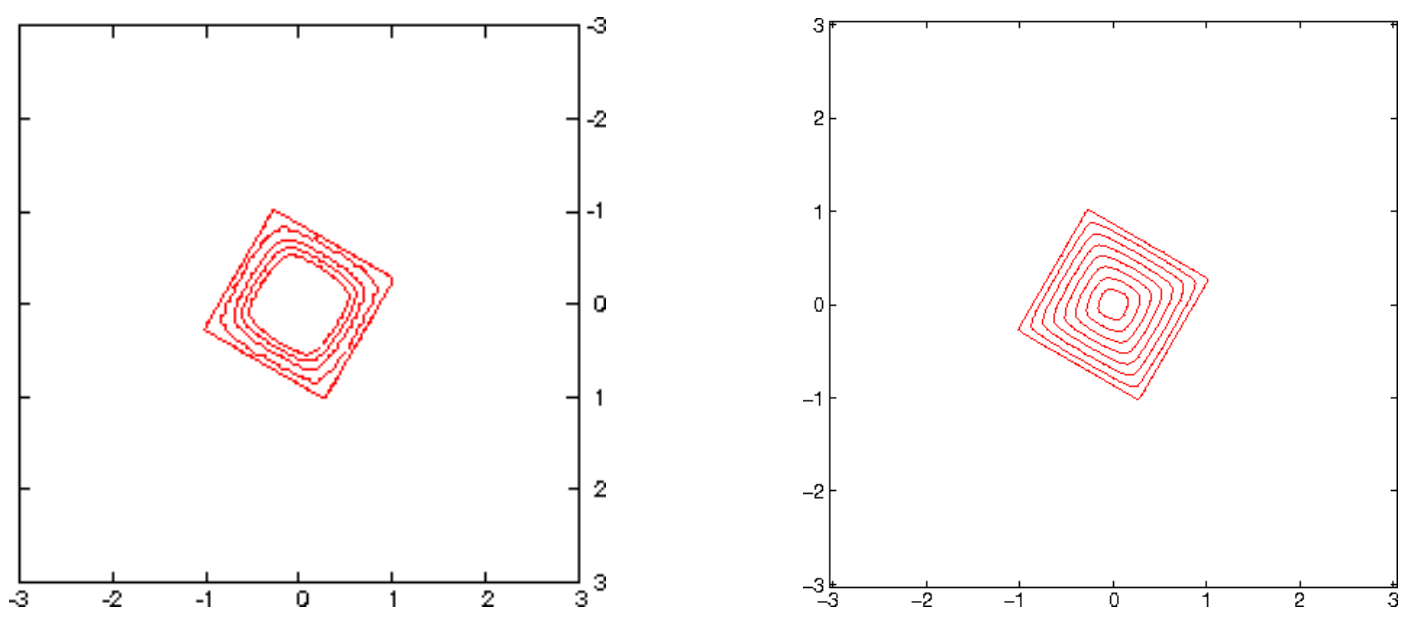

Figure 5: Shrinking of a square approaching a circle with the Level Sets method (left) and GFMM (right) 


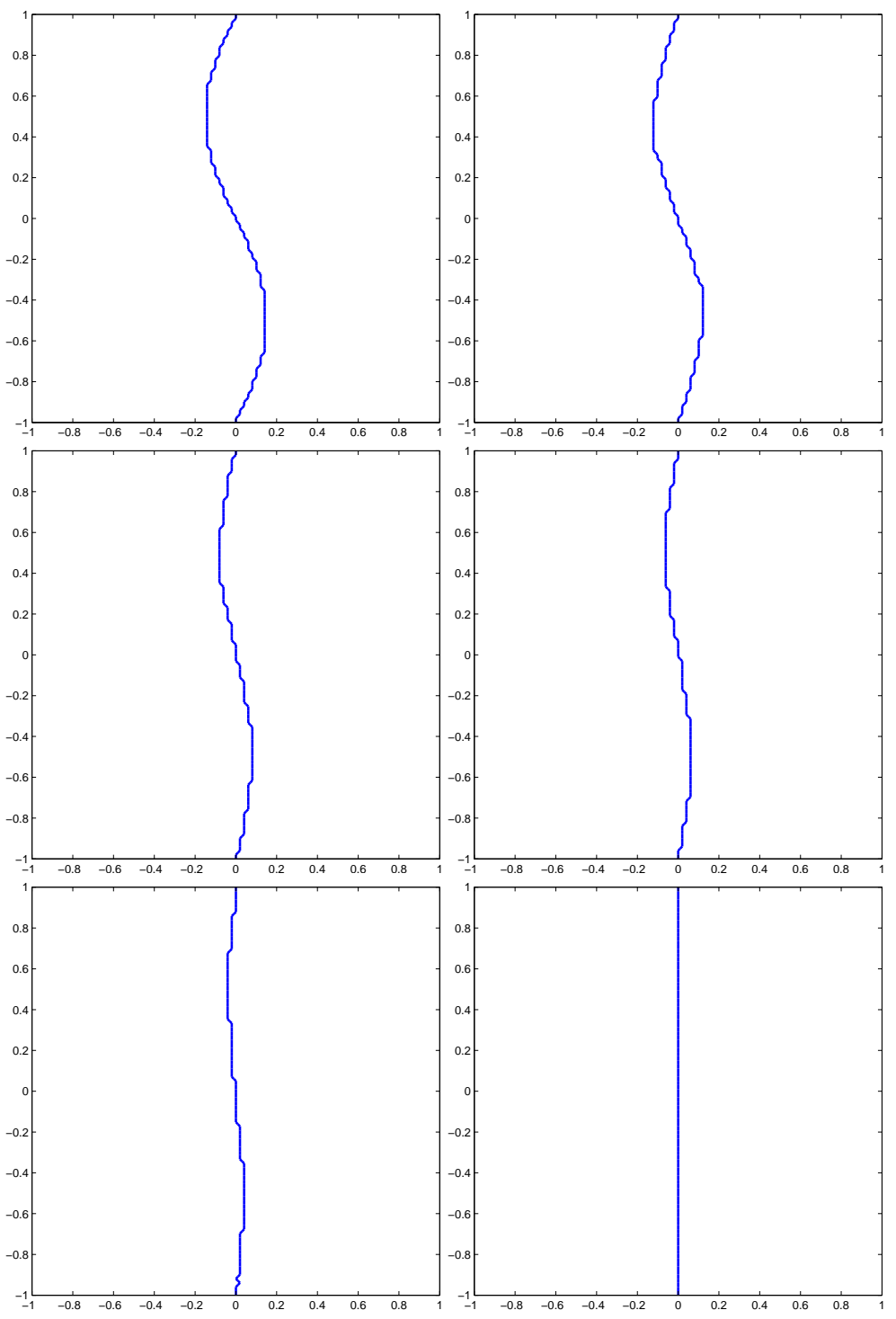

Figure 6: Shrinking of a sinusoidal line dislocation 


\section{A Algorithm used in the numerical tests}

In our tests we have used a slightly modified version of the GFMM algorithm described in Subsection 2.2.

There are two main differences, the first one concerns the time $u_{J \rightarrow I}$. As in [6], we take the same time $u_{J \rightarrow I}$ in all the direction and we denote it by $u_{J}$.

Secondly, since the algorithm described in Subsection 2.2 has constant in time speed on each interval $\Delta T$, it is not necessary recompute the values of $\tilde{u}^{n-1}$ on the whole Narrow Band $N B^{n}$ in this time interval: the computation is needed only on the neighbourhood of the accepted nodes.

\section{Initialization}

1. Set $n=1, p=0, t_{0}=0$.

2. Initialize the field $\theta^{0}$ as

$\theta_{I}^{0}=\left\{\begin{aligned} 1 & \text { for } x_{I} \in \Omega_{0} \\ -1 & \text { elsewhere }\end{aligned}\right.$

3. Initialize the time for points $I \in \mathcal{U}^{0}$

$u_{I}^{0}=0$ for all $I \in \mathcal{U}^{0}$

4. Initialize the speed $\hat{c}_{I}^{0}: c_{I}^{0} \equiv c\left[\theta_{I}^{0}\right]$

5. Initialize $\widehat{u}^{0}$ everywhere on the grid

$$
\widehat{u}_{ \pm, J}^{0}= \begin{cases}u_{J}^{0} & \text { for } J \in F_{ \pm}^{0} \cap \mathcal{U}^{0} \\ \infty & \text { elsewhere. }\end{cases}
$$

6. Compute $\tilde{u}^{0}$ on $N B^{0}$

Let $I \in N B^{0}$, then we compute $\tilde{u}_{I}^{0}$ as the solution of the following second order equation:

$$
\begin{aligned}
& \sum_{k=1}^{N}\left(\max _{ \pm}\left(0, \tilde{u}_{I}^{0}-\widehat{u}_{+, I^{k, \pm}}^{0}\right)\right)^{2}=\frac{(\Delta x)^{2}}{\left|\widehat{c}_{I}^{0}\right|^{2}} \quad \text { if } I \in F_{-}^{0} \\
& \sum_{k=1}^{N}\left(\max _{ \pm}\left(0, \tilde{u}_{I}^{0}-\widehat{u}_{-, I^{k, \pm}}^{0}\right)\right)^{2}=\frac{(\Delta x)^{2}}{\left|\widehat{c}_{I}^{0}\right|^{2}} \quad \text { if } I \in F_{+}^{0}
\end{aligned}
$$

where

$$
I^{k, \pm}=\left(i_{1}, . ., i_{k-1}, i_{k} \pm 1, i_{k+1}, . ., i_{N}\right)
$$

\section{Loop}

7. $\tilde{t}_{n}=\min \left\{\tilde{u}_{I}^{n-1}, I \in N B^{n-1}\right\}$.

8. $t_{n}=\max \left(t_{n-1}, \min \left\{\tilde{t}_{n}, t_{n-1}+\Delta t\right\}\right)$.

9. if $t_{n}<\tilde{t}_{n}$ go to 13 .

10. Initialize the new accepted points

$N A_{ \pm}^{n}=\left\{I \in N B_{ \pm}^{n-1}, \tilde{u}_{I}^{n-1}=\tilde{t}_{n}\right\}, N A^{n}=N A_{+}^{n} \cup N A_{-}^{n}$

11. Reinitialize $\theta^{n}$

$$
\theta_{I}^{n}=\left\{\begin{aligned}
-\theta_{I}^{n-1} & \text { for } I \in N A^{n} \\
\theta_{I}^{n-1} & \text { elsewhere }
\end{aligned}\right.
$$

12. Reinitialize $u_{I}^{n}$ for points $I \in \mathcal{U}^{n}$

If $I \in \mathcal{U}^{n}$ then

(a) if $I \notin \mathcal{U}^{n-1}$ or $I \in N A^{n}$, then $u_{I}^{n}=t_{n}$.

(b) if $I \in \mathcal{U}^{n-1} \backslash N A^{n}$, then $u_{I}^{n}=u_{I}^{n-1}$. 
13. Compute the speed $c_{I}^{n}$ :

We set

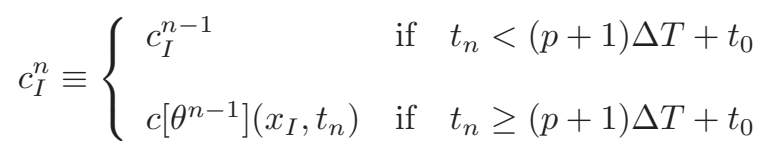

14. Initialize $\widehat{u}^{n-1}$ everywhere on the grid

$$
\widehat{u}_{ \pm, J}^{n}= \begin{cases}u_{J}^{n} & \text { for } J \in F_{ \pm}^{n} \cap \mathcal{U}^{n} \\ \infty & \text { elsewhere. }\end{cases}
$$

15. Compute $\tilde{u}^{n}$ on $N B^{n}$

- If $t_{n}<(p+1) \Delta T$ then we compute $\tilde{u}_{I}^{n}$ on each $I \in N B^{n} \cap V\left(N A^{n-1}\right)$ as the solution of the following second order equation:

$$
\left\{\begin{array}{l}
\sum_{k=1}^{N}\left(\max _{ \pm}\left(0, \tilde{u}_{I}^{n}-\widehat{u}_{+, I^{k, \pm}}^{n}\right)\right)^{2}=\frac{(\Delta x)^{2}}{\left|\widehat{c}_{I}^{n}\right|^{2}} \quad \text { if } I \in F_{-}^{n}, \\
\sum_{k=1}^{N}\left(\max _{ \pm}\left(0, \tilde{u}_{I}^{n}-\widehat{u}_{-, I^{k, \pm}}^{n}\right)\right)^{2}=\frac{(\Delta x)^{2}}{\left|\widehat{c}_{I}^{n}\right|^{2}} \quad \text { if } I \in F_{+}^{n},
\end{array}\right.
$$

- If $t_{n} \geq(p+1) \Delta T$, then we compute $\tilde{u}_{I}^{n}$ as the solution of (A.1) on each $I \in N B^{n}$ and set $p:=p+q^{*}$ where $q^{*}=\sup \left\{q, t_{n} \geq(p+q) \Delta T\right\}$.

16. Set $n:+1$ and go to 7 .

\section{References}

[1] O. Alvarez, P. Cardaliaguet, and R. Monneau, Existence and uniqueness for dislocation dynamics with nonnegative velocity, Interfaces and Free Boundaries, 7 (2005), pp. 415-434.

[2] O. Alvarez, E. Carlini, R. Monneau, and E. Rouy, Convergence of a first order scheme for a non local eikonal equation, IMACS Journal "Applied Numerical Mathematics", 56 (2006), pp. 1136-1146.

[3] — A convergent scheme for a nonlocal hamilton-jacobi equation, modeling dislocation dynamics, Numerische Mathematik, 104 (2006), pp. 413-572.

[4] O. Alvarez, P. Hoch, Y. Le Bouar, and R. Monneau, Dislocation dynamics: short time existence and uniqueness of the solution, Archive for Rational Mechanics and Analysis, 85 (2006), pp. 371-414.

[5] G. Barles, P. Cardaliaguet, O. Ley, and R. Monneau, Global existence results and uniqueness for dislocation equations, SIAM J. Math. Anal., 40 (2008), pp. 44-69.

[6] E. Carlini, M. Falcone, N. Forcadel, and R. Monneau, Convergence of a generalized fastmarching method for an eikonal equation with a velocity-changing sign, SIAM J. Numer. Anal., 46 (2008), pp. 2920-2952.

[7] M. G. Crandall And P.-L. Lions, Two approximations of solutions of Hamilton-Jacobi equations, Math. Comp., 43 (1984), pp. 1-19.

[8] N. ForCADEL, Comparison principle for a generalized fast marching method, SIAM J. Numer. Anal., 47 (2009), pp. 1923-1951.

[9] D. Rodney, Y. Le Bounr, And A. Finel, Phase field methods and dislocations, Acta materialia, 51 (2003), pp. 17-30.

[10] J. A. Sethian, A fast marching level set method for monotonically advancing fronts, Proc. Nat. Acad. Sci. U.S.A., 93 (1996), pp. 1591-1595. 
[11] _ Level set methods and fast marching methods, vol. 3 of Cambridge Monographs on Applied and Computational Mathematics, Cambridge University Press, Cambridge, second ed., 1999. Evolving interfaces in computational geometry, fluid mechanics, computer vision, and materials science.

[12] J. N. TsitsikLIs, Efficient algorithms for globally optimal trajectories, IEEE Trans. Automat. Control, 40 (1995), pp. 1528-1538.

[13] A. Vladimirsky, Static pdes for time-dependent control problems, Interfaces and Free Boundaries, 8 (2006), pp. 281-300. 